\title{
Functional insights into the infective larval stage of Anisakis simplex s.s., Anisakis pegreffii and their hybrids based on gene expression patterns
}

\author{
C. Llorens' ${ }^{1}$, S. C. Arcos'ㄹ, L. Robertson², R. Ramos ${ }^{3}$, R. Futami ${ }^{1}$, B. Soriano ${ }^{1}$, S. Ciordia ${ }^{4}$, M. Careche ${ }^{5}$, \\ M. González-Muñoz ${ }^{6}$, Y. Jiménez-Ruiz², N. Carballeda-Sangiao', I. Moneo ${ }^{6}$, J. P. Albar ${ }^{4}$, M. Blaxter ${ }^{7}$ \\ and A. Navas ${ }^{2 *}$
}

\begin{abstract}
Background: Anisakis simplex sensu stricto and Anisakis pegreffii are sibling species of nematodes parasitic on marine mammals. Zoonotic human infection with third stage infective larvae causes anisakiasis, a debilitating and potentially fatal disease. These 2 species show evidence of hybridisation in geographical areas where they are sympatric. How the species and their hybrids differ is still poorly understood.

Results: Third stage larvae of Anisakis simplex s.s., Anisakis pegreffii and hybrids were sampled from Merluccius merluccius (Teleosti) hosts captured in waters of the FAO 27 geographical area. Specimens of each species and hybrids were distinguished with a diagnostic genetic marker (ITS). RNA was extracted from pools of 10 individuals of each taxon. Transcriptomes were generated using Illumina RNA-Seq, and assembled de novo. A joint assembly (here called merged transcriptome) of all 3 samples was also generated. The inferred transcript sets were functionally annotated and compared globally and also on subsets of secreted proteins and putative allergen families. While intermediary metabolism appeared to be typical for nematodes in the 3 evaluated taxa, their transcriptomes present strong levels of differential expression and enrichment, mainly of transcripts related to metabolic pathways and gene ontologies associated to energy metabolism and other pathways, with significant presence of excreted/secreted proteins, most of them allergens. The allergome of the 2 species and their hybrids has also been thoroughly studied; at least 74 different allergen families were identified in the transcriptomes.

Conclusions: A. simplex s.S., A. pegreffi and their hybrids differ in gene expression patterns in the L3 stage. Strong parent-of-origin effects were observed: A. pegreffi alleles dominate in the expression patterns of hybrids albeit the latter, and A. pegreffii also display significant differences indicating that hybrids are intermediate biological entities among their parental species, and thus of outstanding interest in the study of speciation in nematodes. Analyses of differential expression based on genes coding for secreted proteins suggests that co-infections presents different repertoires of released protein to the host environment. Both species and their hybrids, share more allergen genes than previously thought and are likely to induce overlapping disease responses.
\end{abstract}

Keywords: Anisakis simplex, A. Pegreffii, Transcriptome, Allergens, Gene expression

\footnotetext{
*Correspondence: anavas@mncn.csic.es

${ }^{2}$ Departamento Biodiversidad y Biología Evolutiva, Museo Nacional de

Ciencias Naturales, CSIC, Calle José Gutiérrez Abascal, 2, 28006 Madrid, Spain

Full list of author information is available at the end of the article
}

(c) The Author(s). 2018 Open Access This article is distributed under the terms of the Creative Commons Attribution 4.0 International License (http://creativecommons.org/licenses/by/4.0/), which permits unrestricted use, distribution, and reproduction in any medium, provided you give appropriate credit to the original author(s) and the source, provide a link to the Creative Commons license, and indicate if changes were made. The Creative Commons Public Domain Dedication waiver (http://creativecommons.org/publicdomain/zero/1.0/) applies to the data made available in this article, unless otherwise stated. 


\section{Background}

Anisakis is a genus of nematodes (Nematoda, Rhabditida, Ascaridomorpha: Anisakidae [1]) which parasitizes marine mammals, fishes, molluscs and crustaceans. Anisakis nematodes complete their life cycle in the stomachs of cetaceans, and less frequently pinnipeds, which become infected after devouring paratenic hosts (fishes, cephalopod molluscs or krill) harboring the arrested infective third-stage larvae (L3) [2]. Humans can also be infected by consuming raw or undercooked fish or cephalopod meat. Although L3 of Anisakis spp larvae are unable to complete their life cycle in humans, after penetration of the human gastrointestinal tract they can cause anisakiasis, a condition characterized by acute gastrointestinal manifestations of epigastralgia, nausea, abdominal pain and diarrhoea [3]. Allergic reactions such as IgE-mediated hypersensitivity, angioedema, urticaria and anaphylaxis are also associated with zoonotic exposure of humans to Anisakis spp. [4]. Fish infected with L3 are treated by freezing, which kills the larvae. However, while infection by live L3 is necessary for many of the symptoms of anisakiasis, humans sensitized to Anisakis may show symptoms after consuming fish that has been correctly frozen, cooked and even processed [5-7]. Many Anisakis spp. proteins maintain their allergenic properties even after thermal treatment [7-9]. Episodes of allergic disease in the absence of recent allergen exposure have also been also described [10].

Anisakiasis is emerging as an important epidemiological problem. More than 20,000 cases of anisakiasis have been reported worldwide since 1960 [11], with higher incidences in areas such as Japan, the Netherlands, France, Spain, Germany and California where raw fish is traditionally or increasingly being eaten. Given the widespread consumption of raw fish in the form of sushi and sashimi, Japan has however the highest prevalence of gastric anisakiasis. In Spain, where Anisakis spp. are considered to be an emerging health problem [6], infection frequency ranks between 0.43 and $22 \%$ in fish that reach the market have been reported, with very high rates of infection in particular species [12].

The allergenic potential of Anisakis spp. resides in their allergome (or full set of encoded allergen products). Anisakis spp. contains at least 15 distinct types of major allergens (Ani s 1 to Ani s 14 and Ani s 11.0201) according to the Allergen Nomenclature site (http://www.allergen.org) $[13,14]$. Up to 28 different proteins described as potential new allergens were identified by proteomic analysis of $A$. simplex s.s. and A. pegreffii [15]. A transcriptomic analysis reported 36 and 39 allergens in A. simplex s.s. and $A$. pegreffii respectively [16].

Morphological taxonomy of Anisakis is based on excretory system and esophageal intestinal region of L3 $[17,18]$ and the morphology of adult males [19]. As sampling of adult Anisakis from within definitive hosts is problematic, and L3 diagnosis is difficult, a molecular taxonomic approach for L3, using the nuclear ribosomal internal transcribed spacers (ITS), has been developed [20]. These markers have revealed both potential additional species diversity within Anisakis as well as recombinant genotypes [21-23]. The genus Anisakis is commonly accepted to include 10 named species: $A$. simplex sensu stricto (s.s.), $A$. berlandi (formerly A. simplex C), A. pegreffii, A. typica, A. ziphidarum, A. physeteris, A. brevispiculata, A. paggiae, $A$. nascettii and $A$. schupakovi. At least 2 additional undescribed species (Anisakis sp. 1 and Anisakis sp. 2) have also been designated from L3 genotypes [24, 25]. The most widely studied species are $A$. simplex s.s., $A$. pegreffii and $A$. berlandi: these constitute the Anisakis simplex sensu lato (s.l.) complex [26-28]. A. simplex s.s. and A. pegreffii have been identified as the main etiological agents of anisakiasis [29-33] but it remains unclear if these 2 species are equally pathogenic.

Importantly, A. simplex s.s. and A. pegreffii are able of hybridizing in the areas where they are geographically sympatric [34, 35] and co-infect the same fish host [36]. The described "species" with recombinant genotypes may be the products of interspecific hybridization [34]. While no fertile adult hybrids have been found, L3 with genotypes suggestive of hybridisation between $A$. simplex s.s. and $A$. pegreffii have been identified [24]. These hybrids may express distinct suites of allergenic and immunoreactive proteins [15].

Here we present a transcriptomic analysis of $A$. simplex s.s., $A$. pegreffii and their hybrids sampled in the same development stage (L3) from the same host (Merluccius merluccius) captured in waters where A. simplex s.s. and $A$. pegreffii are sympatric. We used these data to compare their metabolic profiles, gene ontology profiles and expression patterns. We also present an online database, AnisakisDB, which provides access to the abundant data we have generated.

\section{Methods \\ Taxonomic identification and selection of specimens for RNA sequencing}

Larvae specimens in the same development stage (third stage larvae L3) of Anisakis were obtained from the FAO 27 area of fishing distribution into a general survey carried out by our laboratory. L3 were extracted from hosts following published procedures $[34,37,38]$. L3 were rinsed in $0.9 \%$ saline solution, placed in an antibiotic solution for $30 \mathrm{~min}$ and then rinsed in bi-distilled water for $1 \mathrm{~h}$ before molecular identification and RNA extraction. The larvae were individualized (from each host) and for each specimen the caudal part was used for DNA extraction and PCR amplification for species identification; this caudal part and the rest of the body were 
separately stored at $-80{ }^{\circ} \mathrm{C}$ until required. Species identification in that survey was performed following the taxonomic criteria of $[23,39]$ by using the ITS1 region of the nuclear ribosomal DNA (rDNA). Breifly: individual Anisakis spp. L3 stage juveniles was placed in an Eppendorf tube after previously having a small part of the caudal region removed to allow molecular identification of each individual. DNA was extracted and purified using the Speedtools Tissue DNA Extraction Kit (Biotools) following manufactures instructions. Molecular identification was carried out for each individual using PCR-RFLP. The forward primer A 5'-GTCGAATTCGTAGGTGAACCTGCGGAAG GATCA-3' and reverse primer B 5'-GCCGGATCC GAATCCTGGTTAGTTTCTTTTCCT -3' [39] were used in reactions containing, $10 \mathrm{~mm}$ Tris- $\mathrm{HCl}(\mathrm{pH}$ 8.3), $1.5 \mathrm{~mm} \mathrm{MgCl} 2,50 \mathrm{~mm} \mathrm{KCl}, 200 \mathrm{~mm}$ each of dATP, dCTP, dGTP and dTTP and 1 unit of DNA polymerase (Biotools $B$ and $M$ labs, S.A. Madrid, Spain). Initial denaturalization was carried out for a period of $2 \mathrm{~min}$ at $94{ }^{\circ} \mathrm{C}$ followed by 35 cycles of $94{ }^{\circ} \mathrm{C}$ for $1 \mathrm{~min}, 58{ }^{\circ} \mathrm{C}$ for $1 \mathrm{~min}, 72{ }^{\circ} \mathrm{C}$ for $1 \mathrm{~min}$ followed by a final $7 \mathrm{~min}$ extension at $72{ }^{\circ} \mathrm{C}$. Amplified DNA fragments were digested with the restriction enzymes HhaI and Hinfl (New England Biolabs, Massachusetts, MA, USA) following manufacturer's instructions. Restriction fragments were separated by electrophoresis in Tris- Borate-EDTA (TBE) buffered 2.5\% agarose gel, stained with SYBERsafe and visualized with UV illumination.

\section{Specimens selection for trancriptomics}

The detected hybrids can differ as the species (A. simplex s.s. or A. pegreffii) contributing the maternal and paternal genomes, which can influence their expression patterns. We have used mitochondrial DNA sequences, which are inherited maternally, to differentiate the two types of hybrids. We obtained partial sequences of the mitochondrial cytochrome oxidase subunit 2 (COII) gene [40] from each hybrid and determined its maternal species by comparing the COII sequence with a set of COII sequences obtained from 100 populations of Anisakis, using molecular phylogenetic methods. The COII gene was amplified as detailed in [40]. The obtained sequences were pooled with 41 other Anisakis COII sequences downloaded from GenBank [41], and then aligned using the ClustalW program [42] as implemented in Bioedit [43]. Phylogenetic relationship of populations was obtained by Maximum Likelihood and Bayesian Tree (Evolution Model: GTR + I + G) [44] (Additional file 1) considering as outgroup the sequences of Contracaecum sp. and Toxocara canis. The Bayesian information criterion (BIC) as implemented in jModelTest v2.1.4 [45] selected GTR + I + G $(I=0.4950 ; \mathrm{G}=0.6230)$ as the evolutionary model that best fit the data. The selected model and model parameters were used in the maximum likelihood (ML) analysis performed with PhyML v.3.1 [46].
The robustness of the inferred trees was tested by nonparametric bootstrapping (BP) using 1000 pseudo replicates. Bayesian inference was also performed with MrBayes v.3.2.5 [44], running for $1 \times 10^{7}$ generations (four simultaneous Markov chains; sample frequency 100). Four independent partitioned analyses were performed and checked for stationarity and convergence of the chains with Tracer v1.6 [47]. Three data partitions were analyzed: first, second, and third codon positions. Model parameters were estimated independently for each one of the respective data partitions. Burn-in was set to the first 1.000 .000 generations. The robustness of the inferred Bayesian trees was determined using Bayesian posterior probabilities (BPP; as obtained from majority-rule consensus trees of the post burn-in trees) (provided as Additional file 1).

In order to minimize a possible host effect, the larvae used for the three RNA sequencing experiment were obtained from ten different individuals of the same fish host (European hake, Merluccius merluccius) and when it was possible, from fishes which were parasited at the same time by A. simplex s.s., A. pegreffii and their hybrid haplotypes (labeled by yellow and green colors in Additional file 2).

RNA samples from the 3 groups of selected specimens were pooled and $500 \mathrm{ng}$ of each processed using the Illumina TruSeq RNA sample prep kit v.2 (Illumina) to generate 2 poly(A)-selected libraries, one with small inserts (SI library; size range $=200-325 \mathrm{bp}$ ) and one with large inserts (LI library; size range $=260-840$ ). Paired-end reads $(2 \times 100$ bases) were generated on Illumina HiSeq 2000. A total of 19-23 million pass-filter read pairs were obtained from each library. Raw reads were converted to fastq format using Casava on the BaseSpace platform [48]. Read set qualities were assessed using FastQC [49], and trimmed of adapters and low quality data with Cutadapt [50] and Prinseq [51]. An assembly was produced independently for each species (A. simplex s.s. and $A$. pegreffii) and for the hybrids. The SI and LI libraries trimmed fastq files from each specimen group were assembled de novo using Oases $[52,53]$ using a kmer range of 21-31 and following the specifications indicated in the Oases manual. All the assembly metrics provided in Table 1 were obtained using the Perl script Assemblathon stats [54] with the exception of the number of unigenes, which was provided by Oases.

\section{Sequence annotations}

The transcriptomes of A. simplex s.s., A. pegreffii and the hybrids were annotated using the NCBI tool blastx [55] with an evalue of $10 \mathrm{e}^{-5}$ as cutoff threshold and the NCBI non-redundant protein (NR) and the Eukaryotic Orthologous Groups (KOG) databases [56] as reference subjects. Gene Ontology (GO) terms as well as their evidence code [57] and Enzyme Commission (EC) numbers [58] were 
Table 1 Metrics for de novo assembly transcriptomes

\begin{tabular}{lllll}
\hline Summary & A. simplex s.s & A. pegreffii & Hybrid & Merged \\
\hline Total transcriptome size & $88,007,524$ & $68,071,234$ & $50,568,936$ & $67,459,080$ \\
Unigenes (Loci) & 36,645 & 31,988 & 29,656 & - NA- \\
Transcripts (isoforms) & 121,907 & 91,541 & 76,848 & 75,380 \\
Longest transcript (bp) & 10,774 & 11,724 & 10,798 & 16,240 \\
shortest transcript (bp) & 100 & 100 & 100 & 107 \\
$\%$ transcripts $>$ 1Kb & $20.3 \%$ & $21.7 \%$ & $17.9 \%$ & $28.7 \%$ \\
N50 (bp) & 973 & 1026 & 885 & 1276 \\
L50 (bp) & 25,878 & 19,118 & 16,598 & 15,385 \\
\%A & $30.87 \%$ & $30.25 \%$ & $30.11 \%$ & $30.41 \%$ \\
\%C & $19.60 \%$ & $20.03 \%$ & $20.36 \%$ & $18.64 \%$ \\
\%G & $19.64 \%$ & $19.98 \%$ & $20.49 \%$ & $20.60 \%$ \\
\%T & $29.89 \%$ & $29.74 \%$ & $29.04 \%$ & $29.87 \%$ \\
\%Ns & $0 \%$ & $0 \%$ & $0 \%$ & 0 \\
\hline
\end{tabular}

assigned to annotations using correlation tables among GenBank accessions and GO and EC information. InterPro domain [59] annotations were also annotated using identical methodology. Transcripts that had no informative blastx matches were further used to search the NCBI nucleotide database (NT) using NCBI BLAST tool blastn and identical evalue cut-off. The annotation protocol is automatized as a pipeline cited in the section below "pipelines". Proteins (open reading frames) longer than 75 residues were predicted from transcripts using Transeq from the EMBOSS package [60] and OrfPredictor [61]. Signal 4.1 [62] and TMHMM v.2.0 [63] were used to predict secretory signal peptides and transmembrane regions. Metabolic pathways were retrieved from the KEGG web site [64] using EC annotations as queries.

\section{Reference transcriptome}

Minimus2 from the Amos package [65] was used to merge the 3 assemblies into a merged transcriptome. Remaining redundancy was eliminated using CD-HIT [66] at a similarity threshold of 0.95 . The merged transcriptome after redundancy filter resulted in 75,380 consensus transcripts that were annotated via BLAST as described above for transcriptomes using the NR database.

\section{Differential expression and enrichment analyses}

Bowtie2 [67] was used to map the two RNAseq pair-end libraries sequenced per each Anisakis sample on the merged transcriptome. In all cases over $97 \%$ of the reads were successfully mapped on the reference (data not shown). Next, Corset [68] was used to parse the bam files resulting from the mapping step and extract a cluster file grouping the 75,380 consensus transcripts into 74,751 transcript clusters and a count file summarizing the read counts obtained per each cluster from all pair-end libraries mapped to the reference merged transcriptome. The count file was used as input to EdgeR [69] to perform 3 differential expression tests at the whole-transcriptome level: "hybrids vs. A. pegreffii", "hybrids vs. A. simplex s.s." and "A. pegreffii vs. A. simplex s.s.". The SI and LI pair-end libraries prepared from each sample were considered as technical replicates. Transcripts were considered as differentially expressed when they had a $p$-value $<0.05$ after False Discovery Rate (FDR) correction applied by EdgeR using the Benjamini-Hochberg method [70] (referred as just FDR through the rest of the manuscript). Transcripts clusters were assigned the annotations of their consensus transcripts using the GPRO worksheet [71]. Clusters from transcripts with no NR/NT annotations were considered in differential expression analyses only if they had non-zero read counts in at least 2 of the 3 samples.

GO and metabolic pathway enrichment analyses were performed using GOseq [72] and the results from differential expression as input, following the GOseq manual indications for non-native transcriptomes. Concretely, we used 4 input files: 1) a file summarizing the consensus transcripts found as differentially expressed as a FDR $<0.05$ as a cutoff in differential expression analyses; 2) a file with all assayed consensus transcripts; 3) a file with the GOs or with the metabolic maps associated to the consensus transcripts (depending on the analysis); and 4) a file with the sequence size of each assayed cosensus transcript. A priori, enriched GO terms and metabolic pathways supported by $P$-values $<0.05$ in the resulting Wallenius distribution, were considered as significant. A 5\% FDR correction was performed on the P-values obtained from the enrichment analyses using the Benjamini-Hochberg method to outline the most enriched GOs and pathways. 
With the aim to also asses differential expression of genes encoding for excreted or secreted (ES) compounds read counts of transcript clusters with annotation of secretory leader peptide from Signal-P domain or with annotation of GO terms for extracellular compartments were exported to a new count file. Counts in this file from transcripts sharing the same protein descriptions (from NR annotations) were combined using a custom php script called combine_counts.php that is available in AnisakisDB (see the section below AnisakisDB). We combined information from the Uniprot browser [73] and the literature to filter the transcript set to include only those verified as coding for ES products or with confirmed secretory signal peptides but unknown sub-localization. The final count file had 356 non-redundant protein descriptions associated with genes encoding putative ES products. Three differential expression analyses ("hybrids vs. A. pegreffii", "hybrids vs. A. simplex s.s." and "A. pegreffii vs. A. simplex s.s.") were performed using EdgeR, with the small and large insert size libraries of each transcriptome considered as technical replicates (as above).

A third differential expression study focusing on the distinct families of allergens detected in the transcriptomes of A. simplex s.s., A. pegreffii and the hybrids, was also performed. We retrived 509 allergen sequences belonging to 150 allergen families (AllFams) in fungi, plants and animals from the Uniprot protein knowledgebase [73]. We have also used additional information provided by the AllFam database of allergen families [14], and the WHO/IUIS Allergen Nomenclature Database [13, 14]. These 509 allergen sequences were used to identify (via blastx search) homologs in the merged transcriptome. Nine hundred and thirty seven consensus transcript were identified as potential allergen candidates based on a blast e-value threshold of $2 \mathrm{e}^{-06}$. Using identical methods to those described above for ES protein genes, read counts for these 937 candidates were collated for each AllFam family of allergens in a count file that was then used as input to perform 3 differential expression analyses using EdgeR as above.

\section{Pipelines}

The above described protocols for pre-processing, de novo assembly, annotation and differential expression were executed using the DeNovoSeq and the RNAseq pipelines provided by the GPRO suite [70].

\section{AnisakisDB}

We have reconstructed the transcriptomes of the L3 species A. simplex s.s. and A. pegreffii and their hybrids producing a collection of 290,296 transcripts spanning more than $200 \mathrm{Mb}$. We have also reconstructed a consensus transcriptome of 75,380 consensus sequences. Sequences have been annotated using different databases and classificatory systems (NR/NT, KOG, InterPro, GO,
EC) thus creating abundant material (annotations and other material such as scripts, statistics graphical representations and the differential expression and enrichment files). To house the aforesaid material and make it freely available for readers, we constructed a web-available database, AnisakisDB. The database was programmed using Laravel 5 framework for public client platform and a CodeIgniter 2 framework for server platform, both based on the PHP programming language [74], a MySQL database [75] and an Apache server [76] hosted in a Linux environment. The database portal includes a blast search powered by the NCBI BLAST package [77]. Within AnisakisDB, sequences, annotations and backups of all performed analyses of differential expression and enrichment are distributed in seven sections ("Transcriptomes", "Blast", "Download", "COII markers", "Secretome" and Allergome) managed by an intuitive menu and additional utilities to retrieve or compare sequences and annotations. In addition, within the section "Transcriptomes" we have implemented different web site sections providing access to 5 independent Venn Diagrams created based on the GI, KOG, InterPro, GO, EC annotations used to create the Venn diagram of Fig. 1. In each Venn-based web site, clicking on any intersected number the Venn opens a dia$\log$ summarizing the annotations that correspond to the intersection. The Section "COII markers" is another web site where the COII-based phylogeny inferred for selection of specimens has been graphically implemented, also as a dynamic tree representation. AnisakisDB is freely and online accessible at www.anisakis.mncn.csic.es.

\section{Results and discussion}

\section{Transcriptome de novo assembly and annotation}

We sampled Anisakis L3 from a single ocean region (FAO 27) and fish species (M. merluccius), to avoid issues that might arise from response to different paratenic hosts and minimize other environmental and phylogeographic confounding factors. L3 were identified according the taxonomic approaches of $[23,39]$ by using the ITS1 region from rDNA. The phylogenetic tree of 141 COII sequences from 100 populations plus another 41 sequences obtained from GenBank showed all $A$. simplex s.s and all $A$. pegreffii samples clustering in separate sister clades (Additional file 1). The tree also showed that all COII sequences from hybrids fell into one or another of these two clades, allowing immediate classification of hybrids as to their maternal species. RNA was extracted from pools of 10 A. simplex s.s. 10 A. pegreffi and 10 hybrids (five mothered by A. simplex s.s. and five mothered by $A$. pegreffii). Two paired-end RNA-Seq datasets were generated from each pooled sample, forming technical replicates of the transcriptome. Four transcriptome assemblies were generated, one for each sample (A. simplex s.s., A. pegreffii and hybrids) and one 

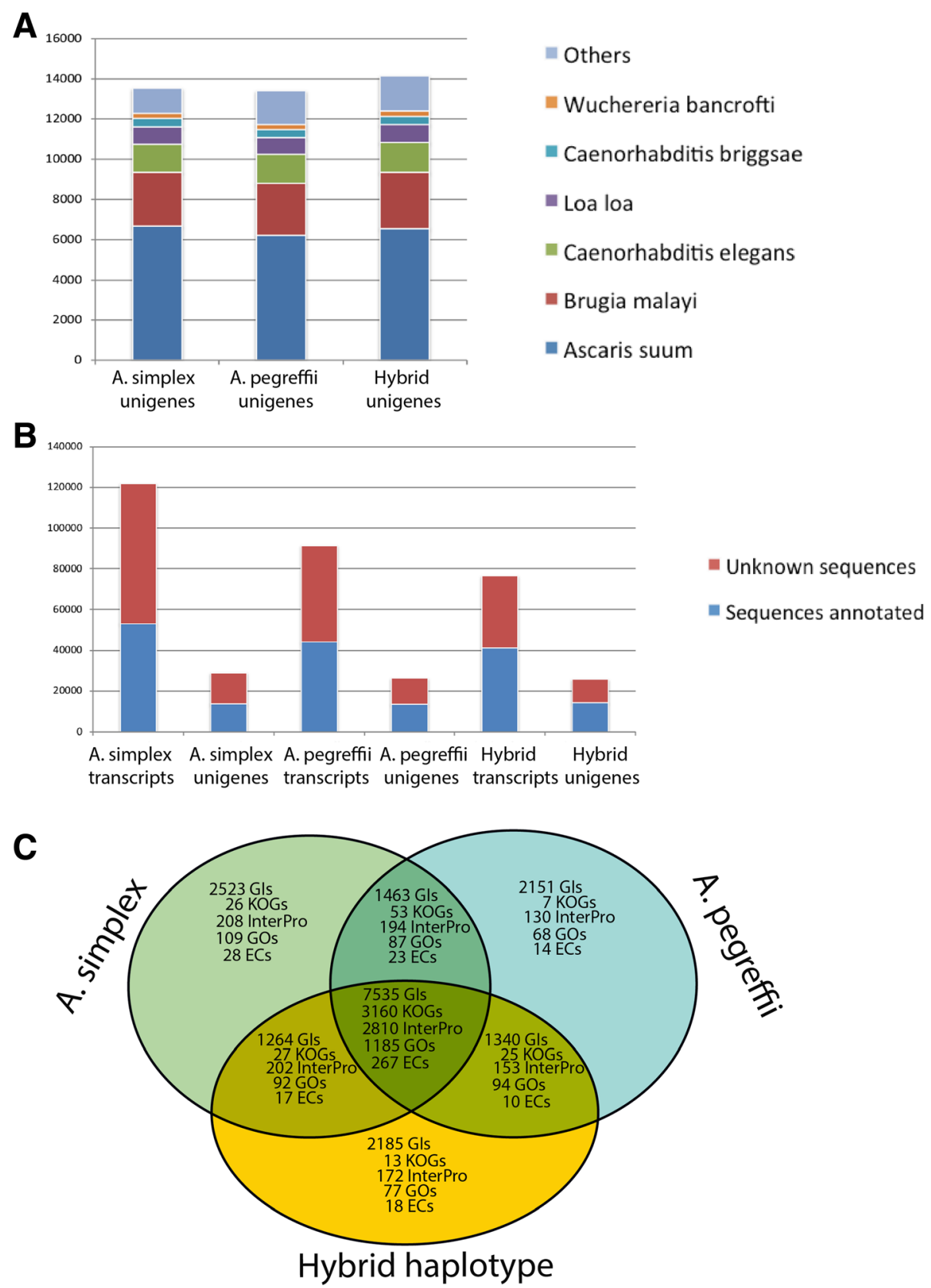

Fig. 1 Annotations. a Blast top hits per subject species using NR as refseq database for unigene annotation. b Distribution of annotated and unannotated transcripts and unigenes annotated via blast using NR as refseq database. c Venn diagram showing unique and shared features of non-redundant annotations per transcriptome (Gls, KOGs, InterPros, GOs, ECs)

that merged the 3 sample-derived ones (Table 1). The 3 sample-derived transcriptomes are similar in span and complexity. The assembly of $A$. simplex s.s. was longer and had an elevated number of predicted transcripts, apparently because of a greater number of isoforms (30,366 more than A. pegreffii assembly and 45,059 more than the hybrid assembly). We think this might be due to sequencing technical issues, elevated levels of alternative splicing (i.e. isoforms) or elevated heterozygosity in the transcriptome of $A$. simplex s.s. as the number of unigenes is in contrast quite equilibrated (4657 unigenes more than A. pegreffii assembly and 6989 more than the hybrid assembly).

\section{Sequence accessions}

$\mathrm{COII}$ sequences have been deposited at European $\mathrm{Nu}$ cleotide Archive (ENA) under the accession numbers LT883269 - LT883368 (Additional file 2) (For more 
details, see the section in results Introducing AnisakisDB). Raw read data have been deposited at the NCBI SRA Study SRP072976, BioProject PRJNA316941 and BioSamples SAMN04592605, SAMN04592630, and SAMN04592599.

The transcriptomes of $A$. simplex s.s., $A$. pegreffii and hybrids were annotated by sequence similarity via blast searches performed against the protein and nucleotide NR, KOG and NT NCBI databases considering an e-value threshold cutoff of $10 \mathrm{e}-5$ and via prediction of secretory signal peptide and transmembrane regions. Annotation features (InterPro domains, GOs, and ECs) were also retrieved from the annotations of protein blast subjects provided by NR database. All annotations are summarized in Table 2. The best hits detected per species in the blast search performed against NR database were provided by proteins of other nematodes such as Ascaris suum, Brugia malayi, and Caernorhabditis elegans (Fig. 1a). Although the transcriptome of $A$. simplex s.s. had the highest number of transcripts, most of these did not detect homologues via blast (Fig. 1b). As it is shown in Table 2, if considering only transcripts with annotation from NR/NT databases, the 3 transcriptomes result in similar number of annotated transcripts (assembled isoforms) and unigenes (loci to which a set of transcripts is assigned). Considering the Gene Identifiers (GIs) to approximate the number of expressed genes we can state that the reconstructed transcriptomes of $A$. simplex s.s., $A$. pegreffii and the hybrids were annotated based on $12,785,12,489$ and 12,324 potential genes, respectively. Annotations thus correct the bias in number of isoforms detected in the assembly of the A. simplex s.s transcriptome, suggesting these sequences might be unknown sequences or potential sequencing artefacts (which for simplicity's sake were not considered in the subsequent comparative analyses). When considering the annotations of the three Anisakis transcriptomes altogether a total number of 18,461 non-redundant GIs were found, which approximate the annotated pan-transcriptome here characterized from A.simplex
S.s., A. pegreffii and their hybrids. Of the 18,461 total GIs, the 3 transcriptomes share 7535 GIs providing additional functional information in the form of 3260 KOG annotations, 2810 InterPro domain annotations, 1185 GO categories and 267 ECs (Fig. 1c). As also shown in that figure, the reconstructed transcriptome of $A$. simplex s.s. shares 1463 GIs with $A$. pegreffii and 1264 GIs with the hybrids. In turn, the hybrids share 1340 GIs with $A$. pegreffii. This therefore suggests that (based on the annotations of these 3 transcriptomes) the hybrid shares at least 8875 GIs with $A$. pegreffii and 8799 GIs with $A$. simplex s.s. In other words, at least 48 and $47.6 \%$ of the annotations of the hybrid transcriptome have homologs in A. pegreffii and A. simplex s.s., respectively (consistently with the hybrid status). A. simplex s.s shares 8998 GIs with A. pegreffii thus meaning that at least $48.7 \%$ of the unigenes annotated in $A$. simplex s.s have homologs in A. pegreffii.

\section{Expression patterns and enrichment of metabolic pathways and gene ontologies}

The merged (consensus) transcriptome reconstructed in this study based on the three Anisakis transcriptome consist of 75,380 consensus sequences, which were used as a mapping reference to compare the expression patterns at the whole-transcriptome level of the 3 taxa. Of the 75,380 consensus transcripts, 32,999 have no annotation and therefore correspond to unknown sequences expressed by the at least two of three taxa (they might be unknown proteins, potential non-coding RNAs and/ or even mobile elements). The remaining 42,381 consensus transcripts correspond to coding sequences annotated based on 12,511 different proteins (according to the number of non redundant GI annotations) thus meaning that any biological interpretation of differential expression and enrichment based on the merged transcriptome is based on the expression patterns of 12,511 coding genes (around 68\% of the pan-transcriptome here characterized based on the three transcriptomes). After mapping the reference transcriptome, the 75,380

Table 2 Summary of annotations per transcriptome

\begin{tabular}{|c|c|c|c|c|c|c|}
\hline \multirow[t]{2}{*}{ Sequences with annotations } & \multicolumn{2}{|c|}{ A. simplex s.s. } & \multicolumn{2}{|l|}{ A. pegreffii } & \multicolumn{2}{|l|}{ Hybrids } \\
\hline & transcripts & unigenes & transcripts & unigenes & transcripts & unigenes \\
\hline Gene Identifiers (Gls) from NR/NT & 62,483 & 14,057 & 60,816 & 14,393 & 53,741 & 14,183 \\
\hline KOG clusters & 38,717 & 9999 & 29,977 & 9444 & 28,114 & 9928 \\
\hline InterPro domains & 17,093 & 5938 & 13,514 & 5122 & 12,884 & 5125 \\
\hline GO Terms & 14,701 & 5165 & 11,773 & 4485 & 11,101 & 4501 \\
\hline ECs & 5416 & 1995 & 4237 & 1671 & 3913 & 1617 \\
\hline Predicted proteins & 49,755 & 14,384 & 41,324 & 13,506 & 38,782 & 13,834 \\
\hline Predicted signal-P domains & 1124 & 647 & 603 & 394 & 482 & 318 \\
\hline Predicted TM domains & 11,613 & 6338 & 8906 & 4676 & 7545 & 4109 \\
\hline
\end{tabular}


consensus sequences of the merged transcriptome were grouped into 74,751 transcript clusters (see Methods) correcting any potential bias in isoforms as detected in the assembly of $A$. simplex s.s. The Biological Coefficient of Variation (BCV) and the average dispersion of the assayed samples were inferred and evaluated to asses to suitability of the fastq libraries, resulting in quite appropriate values $(B C V=0.4359$ and dispersion $=0.19005)$ for a study of differential expression. A similar conclusion resulted from plotting $\mathrm{BCV}$ against the average log of counts per million of mapped reads. Additionally, a multidimensional scaling (MDS) plot based on logFC of the differences among taxa sources and the sequenced replicates per taxa shows that while the SI and LI libraries used as taxon replicates fall close to one another, the replicates of different taxa fall widely separated (Fig. 2b). This result anticipates important differences among species and hybrids. The first plot dimension correspond to the differences among taxa sources (A. simplex s.s., Hybrids, $A$. pegreffii) that provides the major source of variability. The second plot dimension corresponds to the sequenced replicates per taxa (small and medium insert size fastqs) that present practically no differences with the exception of the hybrids where the 2 replicates are slightly more heterogeneous because they were created as a pool of 5 hybrids close to $A$. simplex s.s., and 5 other close to $A$. pegreffii. Consistent with this perspective, of these 74,751 transcript clusters, 8239 resulted as differentially expressed in the analysis performed between the hybrids and $A$. pegreffii using FDR $<0.05$ as cutoff, 23,549 were differentially expressed in the analysis performed between the hybrids and A. simplex s.s. using same FDR, and 24,813 were differentially expressed between $A$. pegreffii and A. simplex s.s. with same FDR (Fig. 2a and Additional file 3). In general, these analyses of differential expression based on the merged transcriptome suggest that the $A$. pegreffii parental expression pattern predominates over A. simplex s.s. in the hybrids, in agreement with population genetic studies of Moroccan Anisakis [36].

The 272 ECs identified as common in the 3 transcriptomes link to 53 metabolic pathways [64] related to nutritional requirements and biosynthesis, energy metabolism, metabolism of xenobiotics, environmental information processing, signal transduction and genetic information processing. Ten of these 53 pathways were shown as differentially enriched in at least one of the 3 enrichment comparisons after correction of the pvalue at FDR $<0.05$ (Additional file 4). As shown in Fig. 3, two of these pathways (oxidative phosphorylation and methane metabolism) were differentially enriched in all comparisons thus indicating that the main metabolic differences among A. simplex s.s., A. pegreffii and their hybrids concern to the energy metabolism. Both $A$. pegreffii and A. simplex s.s. present overexpression with respect to the hybrids as for transcripts associated to methane metabolism while differentially expressed transcripts involved in oxidative phosphorylation are overexpressed in A. pegreffii with respect to the 2 other taxa. Figure 3 also reveals that the enrichment of the glycosaminoglycan degradation pathway was significant between the performed comparisons of the hybrids and $A$. pegreffii against $A$. simplex s.s. but not when the hybrids and $A$. pegreffii are compared. The degradation of glycosaminoglycans pathway mainly concerns to transcripts coding for hyalurononglucosaminidases, which are known to play role in the extracellular matrix as soluble components and polyelectrolytes interacting with growth factors and other transient components of the extracellular matrix [78]. In this case, the enrichment derives from a strong overexpression of the transcripts coding for these enzymes in the hybrids and $A$. pegreffii with respect to A. simplex s.s. Reversely, the pathway of alanine aspartate and glutamate metabolism is shown as significantly enriched when the hybrids and A. simplex s.s. are compared with $A$. pegreffii but not when they were compared each to other thus suggesting that the $A$. pegreffii parental does not predominate in all expression patterns of hybrids. The remaining pathways (pyruvate metabolism, aminoacyl-tRNA biosynthesis, streptomycin biosynthesis, starch and sucrose metabolism, pentose phosphate pathway, and cysteine and methionine metabolism) were found as differentially enriched only in 1 of the 3 performed comparisons, predominantly when $A$. pegreffii was compared against any of the 2 other taxa (Fig. 3). Results sugest that $A$. pegreffii and the hybrids exhibit for these pathways more differentially expressed transcripts than those that would be expected by chance. In fact, the ratio between differentially expressed and assayed transcripts associated to the enrichment of each pathway is predominantly lower when the hybrids are compared with $A$. pegreffii than when they are compared with A. simplex s.s. or when A. simplex s.s. is compared with A. pegreffii. Finally, it is worth to also discuss the detection of streptomycin biosynthesis pathway, which is associated with the over expression of 7 transcripts encoding for 2 type of enzymes, inositol-3-phosphate synthases (lyases) and dTDP-4-dehydrorhamnose 3,5-epimerases (isomerases) in A. pegreffii and A. simplex s.s. when compared to the hybrids. The expression of the genes coding for these enzymes might have been induced by the antibiotic treatment applied on L3 specimens before the extraction (see methods) thus apparently revealing some deficiencies in the hybrids with respect to their parents of for this metabolic pathway. However, we should to also take into account that Inositol-3-phosphate synthase also participates in the metabolism of inositol phosphates, mainly to produce membrane receptor playing role in signalling, cell 

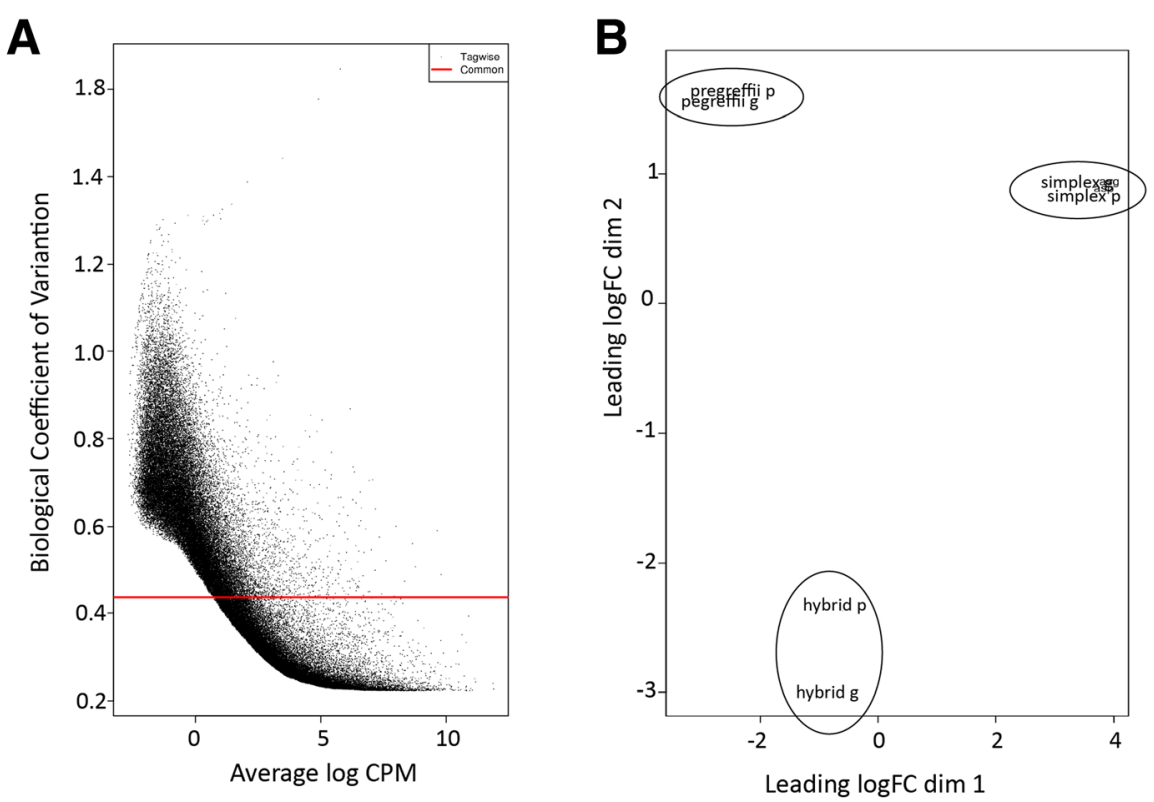

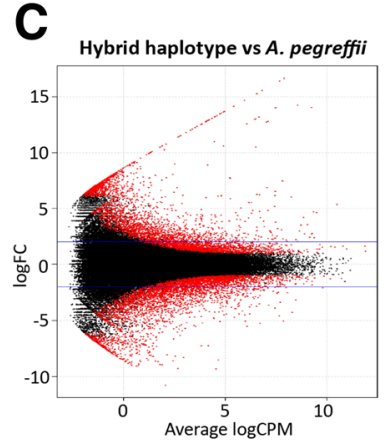

Over expressed in A. pegreffii (4217) Not significant (66512) Over expressed in Hybrid (4022)

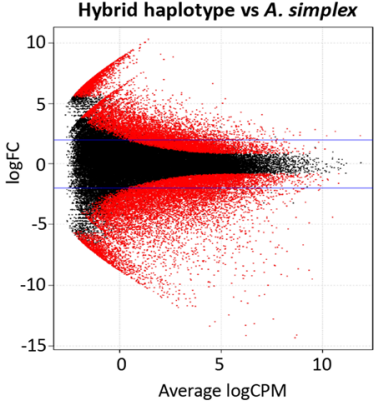

Over expressed in A. simplex (12549) Not significant (51202) Over expressed in Hybrid (11000)

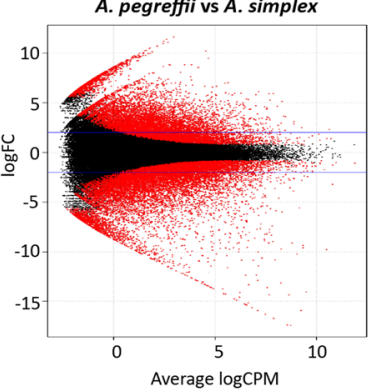

Over expressed in A. simplex (13339) Not significant (49938) Over expressed in A. pegreffii (11474)

Fig. 2 Transcriptome differential expression patterns. a BCV versus the average log of counts per million (CPM). b MDS plot based on logFC of differences among taxa sources and the sequenced replicates per taxa (small and medium insert size fastqs). c MA-plots (one per each differential expression analysis) representing the log Fold Change (logFC) against the log-average log CPM per each transcript cluster across each pair of compared samples. Differentially expressed clusters supported by FDR $<0.05$ are plotted in red. Each MA-plot is accompanied by a summarization of the results of each differential expression comparison

growth, apoptosis, cell migration, endocytosis, and cell differentiation [79], while dTDP-4-dehydrorhamnose 3,5-epimerases are also associated to the nucleotide sugar metabolism and with the biosynthesis of dTDP-rhamnose to produce rhamnose. The biosynthesis of dTDP-rhamnose is a new pathway involved in hypodermal development or in the production of the cuticle or surface coat in larval stages of $C$. elegans and other nematodes [80]. We thus think that inositol-3-phosphate synthases and dTDP-4-dehydrorhamnose 3,5-epimerases of anisakids might link not only to the metabolism of secondary metabolites but also to signalling, nucleotide sugar metabolism and larval development.

Analyses of GO enrichment were also performed based on the 1211 GOs shared by the 3 transcriptomes and revealed differential enrichment of 91 GOs at FDR $<0.05$ in at least on of the 3 performed comparisons (Additional file 4). Forty of these 91 correspond to biological processes terms, 37 are molecular functions and 14 cellular components (Fig. 4). Thirteen of these 91 were significant in all comparisons and correspond with functions, processes and sub-localizations associated with the energy metabolism and this mainly includes oxidases, oxidoreductases, dehydrogenases and other enzymes and binding proteins with assigned roles in redox processes, and electron carriers probably related to the transmembrane electrochemical gradient as well as roles for heme-biding proteins (Cytochrome P450s). We think that the latter might also be related with sterol and heme recruitment. As other nematodes, anisakids probably 
A differentially enriched pathways
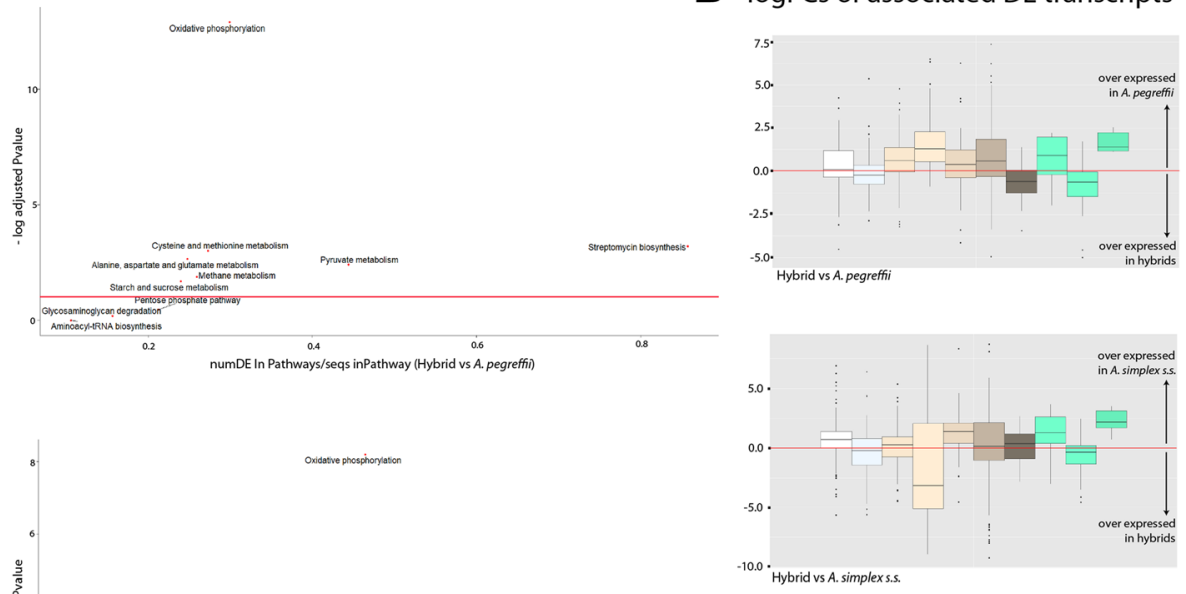

logFCs of associated DE transcripts

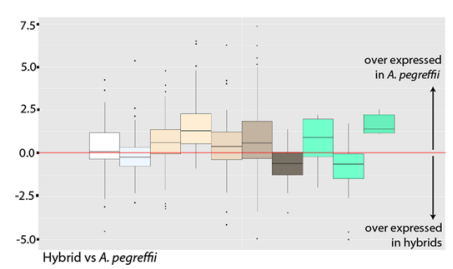

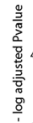
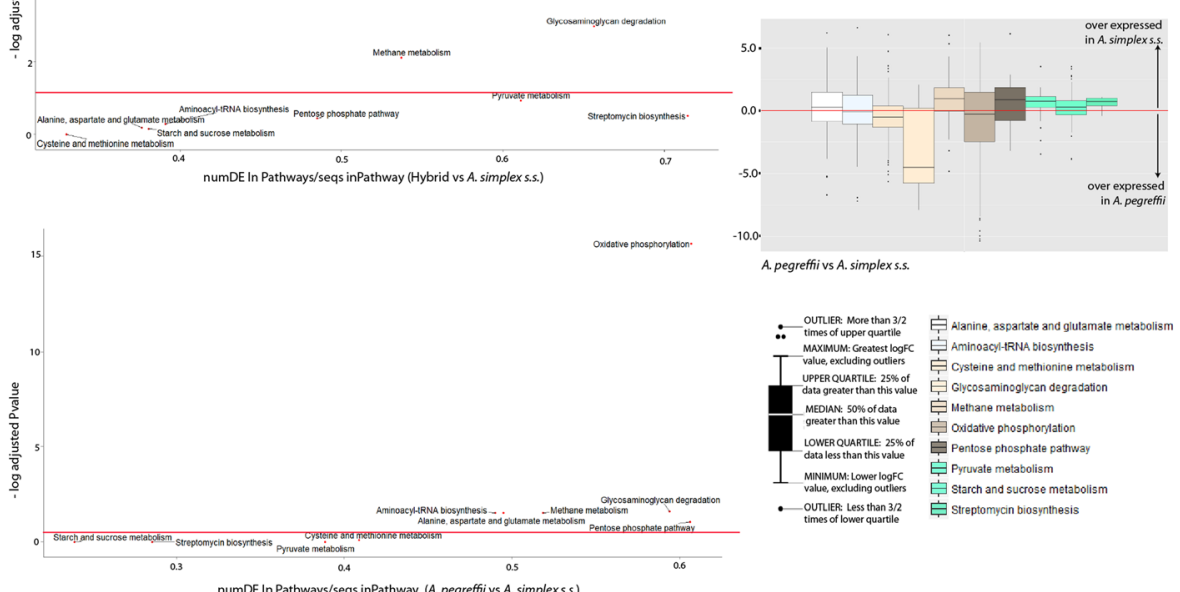

A. pegreffivivs. simplexs.s.

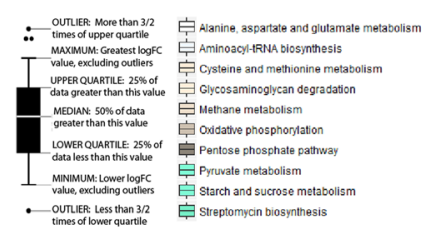

Fig. 3 Enrichment of metabolic pathways. a Scatter plot for the enrichment of metabolic pathways per differential enrichment comparison at FDR $<0.05$. In the $x$-axis, the ratio between number of DE transcripts and total number of transcripts per pathway using the Wallenius approximation; in the $y$-axis, the $\log P$-value for differential enrichment in each pathway. $\mathbf{b}$ Box plots based on the median, quartiles, maximum and minimum and outlier values of the LogFCs of all transcripts contributing to the enrichment of the metabolic pathways shown in $A$ in each differential expression comparison. Red lines highlight the logFC value of zero

recruit exogenous sterol and heme recruited from host sources to implement sterol and heme dietary needs. Consistent with this argument, the 3 transcriptomes addressed in this study present annotations of prenyltransferases, squalene oxidases and sterol regulatory element-binding proteins (SREBP); prenyltransferases play role in the synthesis of pyrophosphate and geranylpyrophosphate, a major metabolic chokepoint in de novo sterol biosynthesis pathway [81] while squalene and derivatives are the main source of host sterol and SREBP is known to stimulate sterol biosynthesis. The enrichment of the remaining GOs shown in Fig. 4 was significant in 1 or 2 of the 3 performed comparisons and refers to molecular functions biological processes and cellular components related to the enriched pathways shown and discussed above but also with transposition (mainly integrases and proteases encoded by $\mathrm{Bel} / \mathrm{Pao}$ LTR retrotransposons), transport, growth and development, locomotion, and with the extracellular region (ES proteins, allergens and cuticle collagens). In broad terms and for almost all evaluated GOs (with several exceptions) the ratio between differentially expressed and assayed transcripts is again lower if the hybrids are compared with A. pegreffii than when the hybrids are compared with A. simplex s.s. or when the latter is compared with $A$. pegreffii. In turn, the comparison between the hybrids and $A$. pegreffii presents largely more enrichment than the others; of the 91 GOs shown as differentially enriched, 67 terms are significant in the aforesaid comparison between the hybrids and A. pegreffii while 27 GOs are significant when the hybrids and A. simplex s.s compared and 34 when the latter is compared with $A$. 


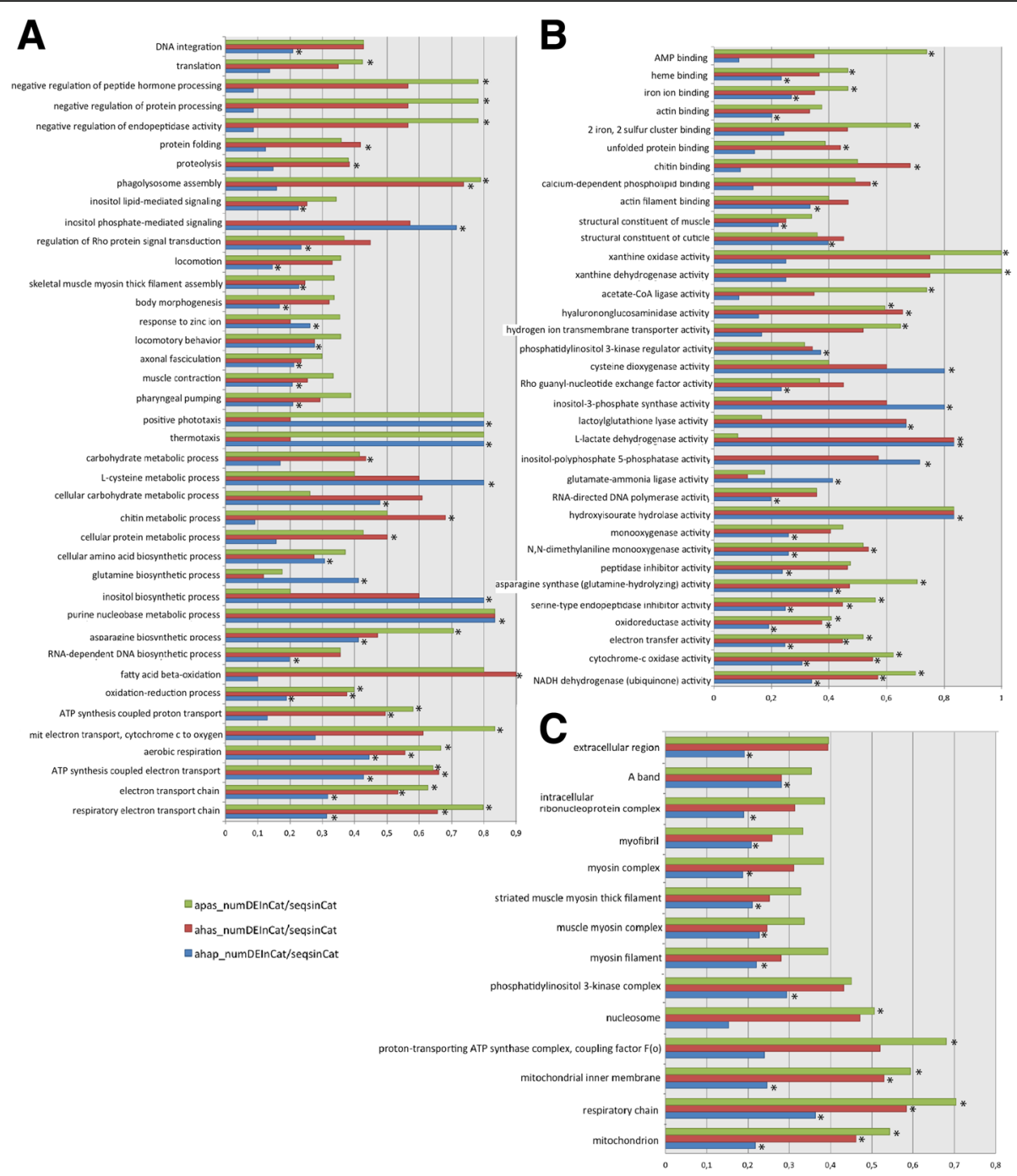

Fig. 4 Differential enrichment of Gene Ontologies. a Barplot based on the ratio between the number of differentially expressed transcripts and the assayed transcripts with annotation of biological process GOs detected as differentially enriched in at least 1 of the 3 comparisons performed to asses $\mathrm{GO}$ enrichment. Plots based on the comparison performed between the hybrids and A. pegreffii is colored blue; plots for the comparison between the hybrids and A. simplex s.s. are colored red; and those based on the comparison between A. pegreffii and A. simplex s.s. are colored green. GOs that were significant at FDR $<0.05$ are highlighted with an asterisk. b Same Bar plot representation based on Molecular Function GOs; c Cellular components

pegreffii. Interestingly, the most prominent differences in GO enrichment observed between the hybrids and the 2 parents derive from the differential expression of transcripts related with the inositol polyphosphate 5-phosphatase activity involved in inositol phosphate-mediated signaling. While these $2 \mathrm{GO}$ terms are significantly enriched when they are compared with their parental species but no when the parents are compared each to other. Indeed, we think that the deregulation of the inositol polyphosphate 5-phosphatase enzyme and how it affects the whole inositol phosphate-mediated signalling pathway it is worth to be investigated, moreover if we take into account that hybrids are not able to reach adult stages and that inositol phosphate-mediated signalling has been associated to longevity, growth and reproduction in nematodes and other invertebrates [82]. The enrichment of GOs is also consistent with the perspectives provided by the differential expression analyses and the enrichment of metabolic pathways that together supports the idea of that although the alleles of the A. pegreffii parental apparently predominate over those of A. simplex s.s. in the expression patterns of L3 larval stage hybrids the latter are intermediate biological entities with metabolic and functional requirements, quantitatively different to those of the two parental species.

The set of metabolic pathways and GOs (i.e. the functional profiles) of A. simplex s.s., A. pegreffii and their 
hybrids are consistent with the aerobic intermediary metabolism of nematodes thus supporting the idea that almost all pathways typically observed in C. elegans and other nematodes are present also in these anisakids. A graphical idealisation of the intermediary metabolism of nematodes is represented in Fig. 5 according to [81-85] and the most representative pathways and GOs detected in the 3 Anisakis transcriptomes have been highlighted. Our conclusion is that the $A$. pegreffii parental predominates over $A$. simplex s.s. in the expression of hybrids albeit the latter, and $A$. pegreffii also present significant differences between them indicating that hybrids are intermediate biological entities with metabolic and functional requirements quantitatively different to those of their parental. The hybrids thus offer an excellent opportunity to investigate the mechanisms of speciation in Anisakis including the novel biological and ecological processes where hybrid genotypes might have a relevant role or significant lacks. Hybridization among $A$. simplex s.s. and $A$. pegreffii has indeed an important role in the evolutionary biology of these 2 species through gene introgression [86]. While hybrids do not appear to reach the definitive host, allowing development to reproductive adults, they reflect the dynamics of moving areas of sympatry. Hybrids may thus be crucial in understanding the microevolutionary processes active in $A$. simplex s.l., in host-parasite ecology (including the range of paratenic hosts exploited), and in whale migrations (as interbreeding must take place in whales as final host).

\section{Genes encoding for excretory/secretory proteins}

To specifically focus on the potential sub-localizations of the products encoded by differentially expressed transcripts associated to enrichment we created a box plot based on the $\operatorname{logFCs}$ of transcripts associated to enriched cellular component GOs (Fig. 6). The analysis shows the relationships of over/under expression observed among the 3 taxa with respect to products sub-localized in mitochondrion, respiratory chain, ATP transporting factors and myosin complexes with ATP-dependent roles in muscle contraction, motility and the extracellular region. A significant number of outliers (due to their extremely high $\log \mathrm{FCs}$ ) correspond to transcripts whose product is sub-localized in the extracellular region thus indicating relevant differences with respect to genes encoding likely

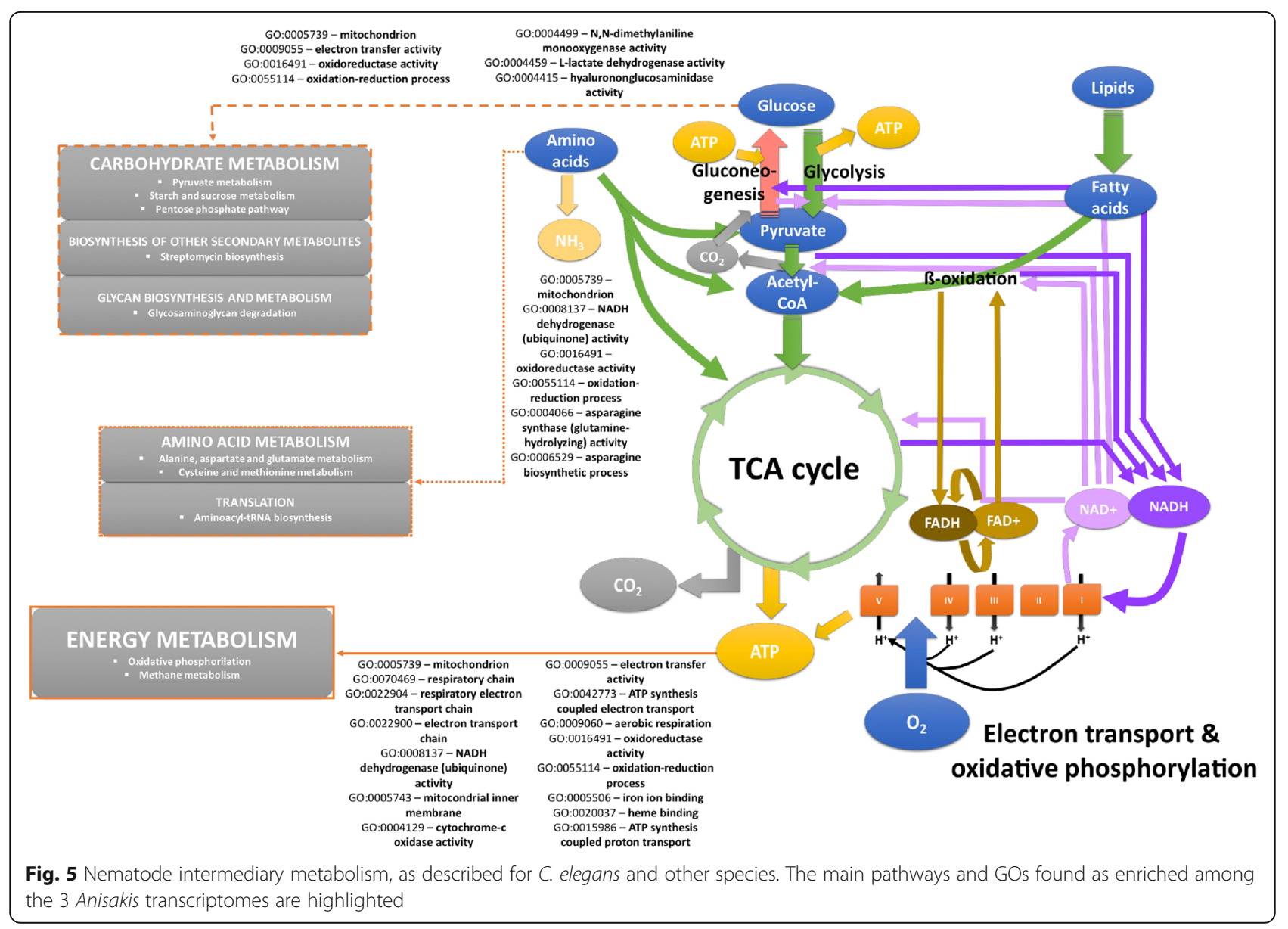




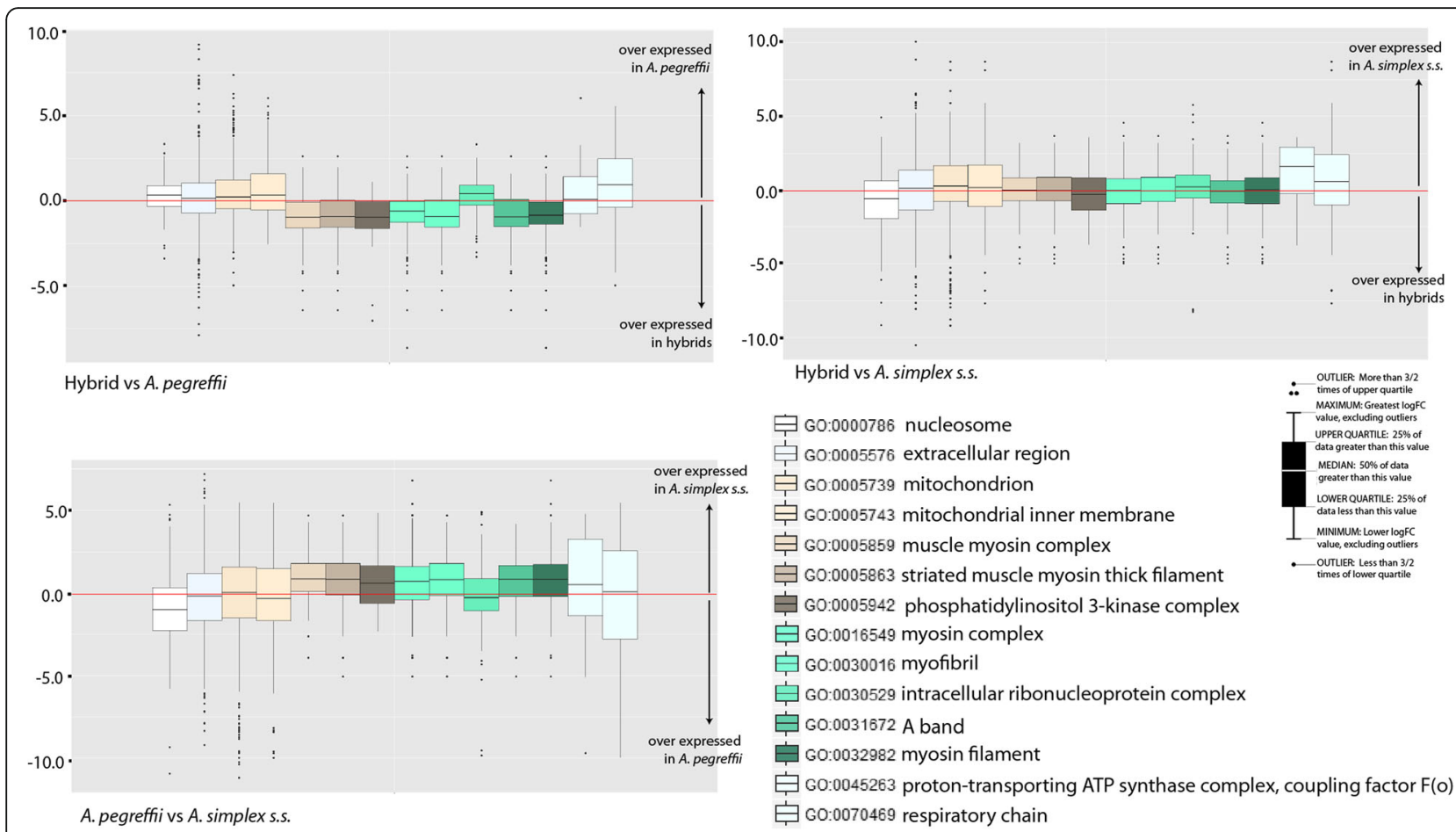

Fig. 6 LogFCs for differentially enriched Cellular Component GOs. Box plots based on the median, quartiles, maximum and minimum and outlier values of the LogFC of all transcripts contributing to the enrichment of Cellular Component GOs per differential expression comparison. Red lines highlight the logFC value of zero

ES proteins. All ascaridids, Anisakis included, release ES products to the host environment. Infective L3 penetrate the intestinal wall of the fish host, and may encyst on the surface of the internal organs or migrate to the muscular tissues. The migration may occur not only when the host is alive [87-91] but also after death [92-94]. These processes are likely to be mediated by secreted enzymes. ES proteins have additional functional roles connected to nutrition, infectivity, allergy, immune evasion or pathogenicity $[34,95,96]$ playing important roles in the host-parasite interaction. In the aforeshown analyses performed at the whole-transcriptome level we showed that part of the differential enrichment between taxa corresponds to transcripts encoding likely ES proteins (above). According to our annotations these transcripts correspond to the expression of at least 356 non-redundant gene descriptions of which we create a count file of mapped reads to explore how different are the expression patterns of the investigated taxa with respect to ES genes (see Methods). Nearly three-quarters $(266 / 356)$ were found to be significantly differentially expressed in one or more comparisons under a FDR $<0.05$ as threshold cutoff (Additional file 5). More specifically, 151 genes were found as differentially expressed when comparing hybrids and $A$. pegreffii. Of these, 79 genes were overexpressed in hybrids and 72 overexpressed in A. pegreffii. Comparing hybrids with $A$. simplex s.s., identified 191 differentially expressed genes, of which 107 were overexpressed in hybrids and 84 in $A$. simplex s.s. Comparing $A$. simplex s.s. and A. pegreffii, 100 transcripts were overexpressed in $A$. pegreffii and 82 in $A$. simplex s.s. A heatmap with clustering of the $\operatorname{logFC}$ per comparison of the 70 most differentially expressed ES genes (Fig. 7) permits to visualize four major patterns of expression. Rows (i.e. the gene expression patterns) split in 4 Clusters of expression designated as clusters 1,2,3 and 4. Cluster 1 groups genes over-expressed in $A$. pegreffii compared to both $A$. simplex s.s. and hybrids, cluster 2 is a single gene, overexpressed in hybrids. Cluster 3 is defined by genes over-expressed in A. simplex s.s. compared to both $A$. pegreffii and hybrids, and genes belonging to Cluster 4 were predominantly over-expressed in both A. pegreffii and hybrids compared to A. simplex s.s. ES genes with allergenic properties are highlighted with red asterisks. In particular, the analysis shows that in mixed infections different species and hybrids of Anisakis may produce different kinds and levels of ES proteins, or in other words that the modulation of the host environment may depend on the mix of larvae from species and hybrids. Considering that hybrids are not able to complete their life cycle and that no colony of L3 hybrids has been yet described in nature parasiting alone their hosts (as far as we know hybrids have been only reported accompanied by their parental species) it is tempting to speculate based on our results that hybrids might be able to survive in host 


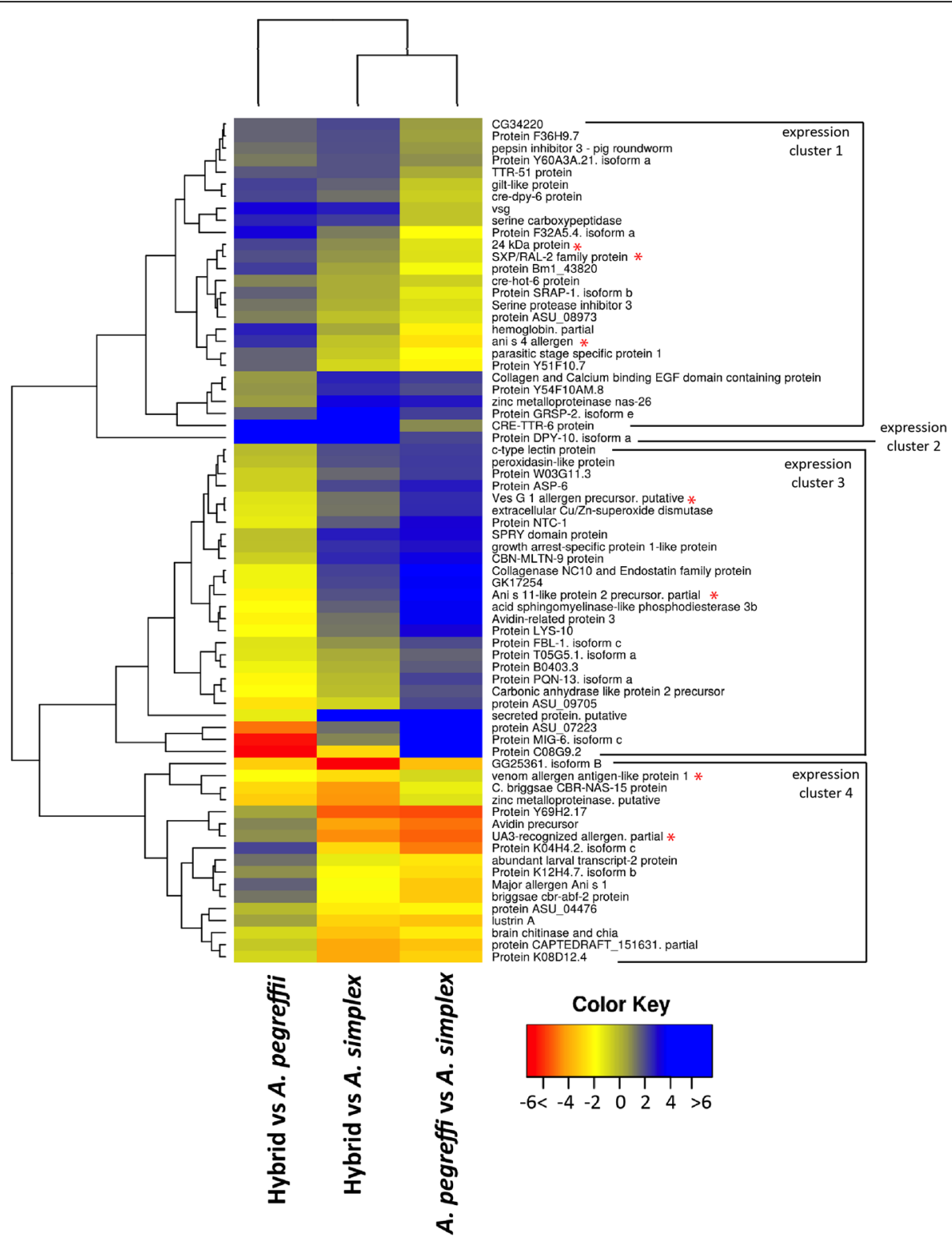

Hybrid vs $A$. pegreffii

Over expressed in $A$. pegreffii (72)

Not significant (205)

Over expressed in Hybrid (79)
Hybrid vs $A$. simplex

Over expressed in A. simplex (84)

Not significant (165)

Over expressed in Hybrid (107)
A. pegreffi vs $A$. simplex

Over expressed in $A$. simplex (82)

Not significant $(174)$

Fig. 7 Expression patterns of excretory/secretory genes. Heatmap with double clustering based on the LogFCs of 70 representative ES genes (rows) identified as differentially expressed at a FDR $<0.05$ in at least 2 of the 3 differential expression comparisons (columns). Quantiles $0.05,0.5$ and 0.95 were used as breaks to color the transcript counts in red, yellow and blue, respectively. Below is, the color scale used to color the gradient of LogFC values. Columns and rows were clustered using the complete linkage with euclidean distance measure. For a logFC summary of the 356 genes assayed see Additional file 5

environments only when they are accompanied by the parental species. Facilitation may also derive from genes overexpressed or downexpressed in the hybrids.

\section{Detection and classification of allergens}

Anisakiasis is a consequence of allergic reaction to several allergenic molecules expressed and/or secreted by
Anisakis in their intermediate hosts. Briefly, 15 allergens (Ani s 1 to Ani s 14 and Ani s troponin C) are formally considered from A. simplex s.s. according to the AllFam database of allergen families and the WHO/IUIS Allergen Nomenclature Database [13, 14]. Five of the 15 Anisakis formal allergens - Ani s1, and Ani s 2 and Ani s 3, Ani s 7, and Ani s 12 - are considered to be major 
allergens (one to which $>50 \%$ of allergic patients immunologically react and present unusual levels of IgE and IgG in serum [97, 98]); Ani s 1 belongs to the animal Kunitz serine protease inhibitor family of protease inhibitors (AF003); Ani s 2 is a myosin heavy chain family (AF100) [99] that includes the highly cross-reactive mite paramyosin major allergens [100]; Ani s 3 is part of the tropomyosin family (AF054), which have been identified as minor inhalative allergens in arthropods and as major food allergens in crustaceans, mollusks and Anisakis [101]; and Ani s 7 and Ani s 12 are 2 proteins with unknown biological functions. The remaining Anisakis allergens are minor allergens; Ani s 4 belongs to the cystatjn family (AF005) [102] of reversibly binding cysteine protease inhibitors; Ani s 6 belongs to the cysteine-rich trypsin inhibitor-like domain family [103] (AF027); Ani s 5, Ani s 8 and Ani s 9 are members of the nematode-specific SXP/ RAL-2 family (AF137), which have unknown function [104, 105]; Anis s 10, Ani s 11 and Ani s 14 similarly remain functionally unclassified [106-108]; Anis s troponin $\mathrm{C}$ is a member of the EF hand family (AF007), which has roles in calcium signaling and binding [109]; and Ani s 13 belongs to the globin family (AF009) [110]. Yet more interesting, recent proteomic analyses have identified up to 28 potential new allergens in Anisakis that can be classified into distinct allergen families [15] and a more recent transcriptomics study [16] has identified several new allergens from A. simplex s.s. and A. pegreffii. Additional potential allergens (Ani s $24 \mathrm{kDa}$, Ani s CCOS3, Ani s cytochrome B, Ani s FBPP, Ani s NADHDS4L, Ani s NARaS and Ani s PEPB) have been also considered according to previous studies [111-113]. All these molecules are classified or can be classified as food allergens because they are present in marine fish. The characterization of allergens in anisakids is still open and that will aid diagnosis and possible alleviation of the serious symptoms of anisakiasis. A complete allergome (all allergen proteins) catalogue for anisakids is required.

Special importance should be paid to proteins related to mechanisms of parasitism and pathogeny of these nematodes. According to a recent transcriptomic study [114] in the pharingeal region of A. simplex s.s. there are at least 226 transcripts which are potentially involved in invasion and host pathogen interplay. The same study found at least 339 transcripts with a similar role in $A$. pegreffii. Regarding this, 13 immunoreactive proteins from the esophageal glands of $A$. simplex s.s. have been described as potential allergens (data not published; PARASITE project [115].

We created a protein sequence database including 509 non-redundant food allergens from fungi, plants and animals (see Methods). This database was used to search homologues in the Anisakis consensus transcriptome we had assembled in this study. We detected high-scoring matches (E-values < 1.99E-06) to 937 consensus transcripts from 121 different allergens that can be classified into 74 families of allergens following the criteria of classification contemplated by AllFamDB and WHO/IUIS $13,14]$ and other classificatory criteria [111-113] (Table 3; Additional file 6). Of the 74 identified families, 41 were detected through similarity to allergens described from animals ( 15 correspond to all Anisakis formal allergens), 15 were detected using fungal allergens and 13 using allergens of plants (i.e. some of these had best matches with allergens from fungi and plants). The remaining 5 sequences were identified using allergens present in more than one realm of life. Differential expression analyses revealed the 74 families to be present in the 3 transcriptomes (Additional file 6). Thirty one families were found as differentially expressed (under a FDR threshold of 0.05 ) when comparing hybrids to $A$. pegreffii, 33 families when comparing hybrids to A. simplex s.s., and 36 families when comparing $A$. pegreffii to A simplex s.s. These expression patterns are consistent although more slightly with the other analyses performed by us (the $A$. pegreffii parental genotype slightly predominates over $A$. simplex s.s in the hybrids' expression patterns). It is striking that all 3 taxa express a large number of different allergen types, many more than has been described previously. So the potential allergen repertoire of these anisakids can be expanded. We think that this repertory might be related to (or be able to partially explain) the cross-reaction of anisakiasis patients to allergens from other sources. It is usually assumed that after sensitization by nematode L3, subsequent contact with these same allergens is required to induce an allergenic response [116]. A number of Anisakis allergens are ES proteins [15], with high affinity to specific immunoglobulin E (IgE) [117], and patients primed by A. simplex s.s. might respond adversely to cross-reactive antigens of not only other anisakids but also of other nematodes and arthropods [118]. Somatic allergens such as Ani s 3 (tropomyosin) cross-react with proteins of crustaceans, molluscs and insects (particularly the American cockroach) [119].

\section{Conclusions}

A. simplex s.s., A. pegreffi and their hybrids show differences in their gene expression patterns in the larval L3 stage. Strong parent-of-origin effects were observed indicating that hybrids are intermediate biological entities among their parental species, and thus of great interest in the study of speciation in nematodes. Differential expression analyses based on genes coding for secreted proteins suggests that co-infections present different repertoires of released proteins to the host environment. Both species and their hybrids, share more allergen genes than previously thought and are likely to induce 
Table 3 Distribution of Allergen Families (AllFams) and logFC results from differential expression

\begin{tabular}{|c|c|c|c|c|c|c|c|c|}
\hline Allergen Families & FamlD & $\begin{array}{l}\text { LogFCs } \\
\text { Hh vs Ap }\end{array}$ & $\begin{array}{l}\text { LogFCs } \\
\text { Hh vs As }\end{array}$ & $\begin{array}{l}\text { LogFCs } \\
\text { Ap vs As }\end{array}$ & Con Seqs & Allergen Name & Realm & Type of allergen \\
\hline $24 \mathrm{kDa}$ protein & $\begin{array}{l}\text { Not yet } \\
\text { assigned }\end{array}$ & $2,24,615$ & $0,94,729$ & $-1,29,887$ & 20 & NA & Animal & NA \\
\hline 605 acidic ribosomal protein & AF070 & $0,70,555$ & $0,25,574$ & $-0,44,982$ & 4 & $\begin{array}{l}\text { Asp f 8, Alt a 12, Asp f 8, } \\
\text { Fus c } 1\end{array}$ & Fungi & Minor \\
\hline Aldehyde dehydrogenase & AF040 & 0,06989 & $-0,10,196$ & $-0,17,186$ & 26 & Blo t 4 & Animal & Minor \\
\hline Alpha-amylase & AF033 & $-0,05472$ & $0,32,169$ & $0,37,641$ & 6 & Alt a 10, Cla h 10 & Fungi & Minor \\
\hline Ani s 10 allergen & $\begin{array}{l}\text { Not yet } \\
\text { assigned }\end{array}$ & 1,07632 & 0,06603 & $-1,01029$ & 1 & Ani s 10 & Animal & NA \\
\hline Ani s 11 allergen & $\begin{array}{l}\text { Not yet } \\
\text { assigned }\end{array}$ & $-2,67,162$ & $2,26,799$ & $4,93,961$ & 6 & $\begin{array}{l}\text { Ani s } 11 \text {, Ani s } 11 \mathrm{~L} 1 \text {, } \\
\text { Ani s } 11 \mathrm{~L} 2\end{array}$ & Animal & NA \\
\hline Ani s 12 allergen & $\begin{array}{l}\text { Not yet } \\
\text { assigned }\end{array}$ & $-0,22,028$ & $-0,25,132$ & $-0,03103$ & 4 & Ani s 12 & Animal & Major \\
\hline $\begin{array}{l}\text { Animal Kunitz serine } \\
\text { protease inhihibitor }\end{array}$ & AF003 & $1,50,377$ & $-1,17,032$ & $-2,67,407$ & 9 & Ani s 1 & Animal & Major \\
\hline ARM-like & $\begin{array}{l}\text { Not yet } \\
\text { assigned }\end{array}$ & $0,88,344$ & $-4,73,273$ & $-5,61,615$ & 44 & Ani s 7, Ani s 14 & Animal & Major, NA \\
\hline $\begin{array}{l}\text { ATP:guanido } \\
\text { phosphotransferase }\end{array}$ & AF049 & $0,28,397$ & 0,03604 & $-0,24,793$ & 5 & $\begin{array}{l}\text { Pen m 2, Der p 20, } \\
\text { Plo i } 1\end{array}$ & Animal & Major, Minor \\
\hline ATP synthase & $\begin{array}{l}\text { Not yet } \\
\text { assigned }\end{array}$ & $0,41,629$ & $0,19,251$ & $-0,22,378$ & 3 & Bos d OSCP & Animal & Minor \\
\hline BCL7 family & AF121 & $-0,42,399$ & $0,29,518$ & $0,71,916$ & 1 & Hom s 3 & Animal & NA \\
\hline Calreticulin family & AF055 & $-0,27,705$ & $\overline{0}, 65,631$ & $-0,37,927$ & 7 & Pen ch 31 & Animal & NA \\
\hline CAP family & AF044 & $\overline{0}, 63,714$ & $-1,22,008$ & $-0,58,293$ & 12 & $\begin{array}{l}\text { sol i 3, Dol a 5, Vesp c5, } \\
\text { Poly s 5, Pol d 5, Dol m 5, } \\
\text { allrgn_V5/Tpx1 }\end{array}$ & Animal & Major, NA \\
\hline Carboxylesterase & AF140 & $-0,07113$ & $-0,09074$ & $-0,01961$ & 50 & Api m 8 & Animal & Minor \\
\hline $\begin{array}{l}\text { Casein kinase II regulatory } \\
\text { subunit }\end{array}$ & $\begin{array}{l}\text { Not yet } \\
\text { assigned }\end{array}$ & $-0,54,905$ & $-0,81,674$ & $-0,26,768$ & 2 & Gal d Phosvitin & Animal & $\mathrm{Na}$ \\
\hline Catalase & AF047 & $1,53,912$ & $0,81,466$ & $-0,72,447$ & 1 & Pen c 30 & Fungi & Minor \\
\hline $\begin{array}{l}\text { Chitinase class III and } \\
\text { peritrophin-like }\end{array}$ & AF077 & $-0,55,642$ & $-0,22,104$ & $0,33,539$ & 12 & Der p 15, Der f 15 & Animal & Major \\
\hline Collagen & AF097 & $-0,20,422$ & $0,10,867$ & $0,31,289$ & 2 & Bos d alpha 2 I & Animal & NA \\
\hline Cyclophilin & AF038 & $0,18,030$ & $0,27,158$ & 0,09128 & 13 & Asp f 27 & Fungi & NA \\
\hline Cystatin & AF005 & $2,62,999$ & $-0,32,427$ & $-2,95,425$ & 4 & Ani s 4 & Animal & NA \\
\hline $\begin{array}{l}\text { Cysteine-rich trypsin } \\
\text { inhibitor-like domain }\end{array}$ & AF027 & $-2,05519$ & 0,03836 & 2,09355 & 5 & Anis 6 & Animal & NA \\
\hline Cytochrome b-c1 & $\begin{array}{l}\text { Not yet } \\
\text { assigned }\end{array}$ & $0,59,193$ & $0,94,815$ & $0,35,621$ & 6 & NA & Animal & NA \\
\hline Cytochrome B5 & $\begin{array}{l}\text { Not yet } \\
\text { assigned }\end{array}$ & $1,95,134$ & $0,37,530$ & $-1,57,620$ & 3 & NA & Animal & NA \\
\hline Cytochrome c & AF006 & $0,14,607$ & 1,04446 & $0,89,838$ & 5 & Cur I 3 & Fungi & Major \\
\hline EF hand family & AF007 & $0,20,495$ & $0,40,417$ & $0,19,922$ & 34 & $\begin{array}{l}\text { Bla g 6, Per a 6, Lit v 3, } \\
\text { Ani s Troponin C }\end{array}$ & Animal & $\begin{array}{l}\text { Major, Minor, } \\
\text { NA }\end{array}$ \\
\hline Enolase & AF031 & $-0,52,169$ & $-0,22,686$ & $0,29,484$ & 5 & rho $m$ 1, hev b 9, Alt a 6 & Fungi,Plant & Minor \\
\hline Eukaryotic aspartyl protease & AF004 & $-0,11,780$ & $-0,35,758$ & $-0,23,979$ & 14 & Bla g 2 & Animal & Major \\
\hline $\begin{array}{l}\text { Eukaryotic elongation factor } \\
1\end{array}$ & AF011 & 0,03983 & $-0,05788$ & $-0,09771$ & 8 & Pen c 24 & Fungi & NA \\
\hline $\begin{array}{l}\text { Fe/Mn superoxide } \\
\text { dismutase }\end{array}$ & AF020 & 0,00817 & $0,19,412$ & $0,18,595$ & 2 & Hev b 10 & Plant & Minor \\
\hline Gelsolin family & AF074 & $-0,81,825$ & $-0,61,973$ & $0,19,852$ & 17 & Der f 16 & Animal & Minor \\
\hline
\end{tabular}


Table 3 Distribution of Allergen Families (AllFams) and logFC results from differential expression (Continued)

\begin{tabular}{|c|c|c|c|c|c|c|c|c|}
\hline Allergen Families & FamlD & $\begin{array}{l}\text { LogFCs } \\
\text { Hh vs Ap }\end{array}$ & $\begin{array}{l}\text { LogFCs } \\
\text { Hh vs As }\end{array}$ & $\begin{array}{l}\text { LogFCs } \\
\text { Ap vs As }\end{array}$ & Con Seqs & Allergen Name & Realm & Type of allergen \\
\hline GILT family & AF155 & $1,61,144$ & $0,92,869$ & $-0,68,276$ & 3 & Tri a 27 & Plant & NA \\
\hline Globin & AF009 & $2,89,321$ & $0,55,871$ & $-2,33,450$ & 3 & Ani s 13 & Animal & Minor \\
\hline Glutathione S-transferase & AF010 & 0,06592 & $0,47,962$ & $0,41,370$ & 11 & Bla g 5, Der p 8 & Animal & Major \\
\hline Glyoxalase superfamily & AF082 & $-0,40,931$ & $0,77,379$ & $1,18,310$ & 2 & Ory Glyosalase I & Plant & NA \\
\hline GMC oxidoreductase & AF081 & $0,37,676$ & $0,45,602$ & 0,07926 & 10 & Mala s 12 & Fungi & Major \\
\hline Heat shock protein Hsp70 & AF002 & $-0,17,513$ & $-0,64,037$ & $-0,46,524$ & 45 & Pen c 10, Cla h HSP70 & Fungi & Minor \\
\hline Heat shock protein Hsp90 & AF042 & -0.19335 & 0.11856 & 0.31191 & 15 & Asp $f 12$ & Fungi & NA \\
\hline Hevein-like domain & AF043 & 0.35419 & -0.39541 & -0.74952 & 1 & Hev b 11 & Plant & Minor \\
\hline Histidine acid phosphatase & AF062 & -0.03153 & -0.32299 & -0.29146 & 15 & Api $m 3$ & Animal & Minor \\
\hline Hyaluronidase & AF103 & 1.02016 & -0.32364 & -1.34380 & 10 & Pol a 2, Api m 2 & Animal & NA. Major \\
\hline $\begin{array}{l}\text { Intermediate filament } \\
\text { protein }\end{array}$ & AF008 & -0.30492 & -0.89969 & -0.59477 & 13 & Hom s 5 & Animal & NA \\
\hline $\begin{array}{l}\text { Lactate/malate } \\
\text { dehydrogenase }\end{array}$ & AF014 & -0.78551 & -0.56985 & 0.21566 & 3 & Cit la MDH & Plant & Major \\
\hline $\begin{array}{l}\text { Larval allergen (Brugia } \\
\text { malayii) }\end{array}$ & Unclassified & 0.32808 & 1.48199 & 1.15313 & 2 & NA & Animal & NA \\
\hline Lipase & AF037 & 0.09487 & -0.64847 & -0.74333 & 15 & $\begin{array}{l}\text { Dol m1, Pol d 1, rhi o } \\
\text { Lipase, The I lipase }\end{array}$ & $\begin{array}{l}\text { Animal. } \\
\text { Plant }\end{array}$ & Major. NA \\
\hline Lipocalin & AF015 & 0.51183 & 0.05554 & -0.45629 & 3 & tyr p 13 & Animal & Minor \\
\hline Major allergen (Loa loa) & Unclassified & -0.00868 & -0.22329 & -0.21461 & 4 & NA & Animal & NA \\
\hline Myosin heavy chain & AF100 & -0.21310 & 0.07600 & 0.28910 & 179 & $\begin{array}{l}\text { Ani s 2, Blo t 11, Der f } \\
\text { 11, der p } 11\end{array}$ & Animal & Major. Minor \\
\hline NAC domain & AF107 & 0.58475 & 0.39473 & -0.19002 & 3 & Hom s 2 & Animal & NA \\
\hline Nitrophorin & Unclassified & 0.27507 & 0.31344 & 0.03836 & 5 & Cim I Nitrophorin & Animal & NA \\
\hline $\begin{array}{l}\text { Papain-like cysteine } \\
\text { protease }\end{array}$ & AF030 & -0.01475 & 0.08466 & 0.09941 & 24 & $\begin{array}{l}\text { Act d 1, Ana c 2, car p 1, } \\
\text { Gly m Bd } 30 \text { k, blo t } 2\end{array}$ & Animal.Plant & Major. NA \\
\hline Patatin family & AF104 & -0.27609 & -3.25600 & -2.97961 & 1 & Hev b 7 & Plant & Major \\
\hline Phosphoglycerate kinase & Unclassified & -0.22025 & -0.55632 & -0.33607 & 5 & Can a PGK & Fungi & NA \\
\hline Profilin family & AF051 & 0.19390 & -0.08435 & -0.27824 & 1 & Par j 3 & Plant & Major \\
\hline Prolamin superfamily & AF050 & 0.07050 & -0.07100 & -0.14148 & 1 & Tri a 26 & Plant & Major \\
\hline Prolyl oligopeptidase family & AF061 & -0.04633 & 0.30623 & 0.35255 & 6 & Ves $\vee 3$, Tri r 4 & $\begin{array}{l}\text { Animal, } \\
\text { Fungi }\end{array}$ & Major. NA \\
\hline $\begin{array}{l}\text { Proteasome subunit beta } \\
\text { type }\end{array}$ & Unclassified & 0.98838 & 1.02852 & 0.04012 & 3 & Zea m $20 \mathrm{~S}$ & Plant & Minor \\
\hline Protein kinase & AF017 & -0.43020 & -0.00592 & 0.42428 & 101 & Sal k 2 & Plant & Major \\
\hline Ribosomal protein L3 & AF058 & 1.01034 & 1.22633 & 0.21599 & 2 & Asp $f 23$ & Fungi & Minor \\
\hline SART-1 family & AF116 & -0.01191 & -0.21989 & -0.20797 & 1 & Hom s 1 & Animal & NA \\
\hline $\begin{array}{l}\text { Serpin serine protease } \\
\text { inhibitor }\end{array}$ & AF018 & 0.84445 & 0.38305 & -0.46140 & 12 & Gal d 2 & Animal & Major \\
\hline Short-chain dehydrogenase & AF028 & -0.09468 & 0.02563 & 0.12031 & 19 & Alt a 8, Cla h 8 & Fungi & Major. Minor \\
\hline $\begin{array}{l}\text { Subtilisin-like serine } \\
\text { protease }\end{array}$ & AF021 & 0.06703 & -1.01313 & -1.08002 & 1 & Rho $\mathrm{m} 2$ & Fungi & Major \\
\hline SXP/RAL-2 family & AF137 & 1.79413 & 0.74628 & -1.04786 & 15 & Ani s 8, Ani s 9, Ani s 5 & Animal & Minor \\
\hline Thioredoxin & AF023 & 0.57209 & 0.74939 & 0.17728 & 1 & Asp f 28 & Fungi & Major \\
\hline $\begin{array}{l}\text { Translationally controlled } \\
\text { tumour protein }\end{array}$ & AF136 & 0.40520 & -0.40064 & -0.80584 & 3 & Hom s TCTP & Animal & NA \\
\hline Triosephosphate isomerase & AF032 & 0.01199 & 0.03933 & 0.02734 & 2 & Tri a 31 & Plant & Minor \\
\hline
\end{tabular}


Table 3 Distribution of Allergen Families (AllFams) and logFC results from differential expression (Continued)

\begin{tabular}{|c|c|c|c|c|c|c|c|c|}
\hline Allergen Families & FamlD & $\begin{array}{l}\text { LogFCs } \\
\text { Hh vs } A p\end{array}$ & $\begin{array}{l}\text { LogFCs } \\
\text { Hh vs As }\end{array}$ & $\begin{array}{l}\text { LogFCs } \\
\text { Ap vs As }\end{array}$ & Con Seqs & Allergen Name & Realm & Type of allergen \\
\hline Tropomyosin & AF054 & -0.31997 & -0.39583 & -0.07586 & 17 & $\begin{array}{l}\text { Ani s } 3 \text {, Bal r 1, Pat y } 1, \\
\text { Hal d 1, Hel as 1, Por s 1, } \\
\text { Der p 10, Pan s 1, } \\
\text { Cra g } 1\end{array}$ & Animal & $\begin{array}{l}\text { Major. Minor. } \\
\text { NA }\end{array}$ \\
\hline Troponin I and T & AF146 & 0.23760 & -0.36364 & -0.60124 & 2 & Pon 17 & Animal & Minor \\
\hline Trypsin-like serine protease & AF024 & -0.33264 & 0.37800 & 0.71063 & 4 & $\begin{array}{l}\text { Blo t 3, Api m 7, } \\
\text { Der f } 6\end{array}$ & Animal & Minor. NA \\
\hline Tuber Storage Protein & Unclassified & 0.26964 & 0.69673 & 0.42708 & 5 & Dio o TSP & Plant & NA \\
\hline Tubulin/FtsZ family & AF025 & -0.22307 & -0.26109 & -0.03802 & 46 & $\begin{array}{l}\text { Lep d alpha Tubulin, } \\
\text { Tyr p alpha Tubulin }\end{array}$ & Animal & Minor \\
\hline WD-40 repeat & AF142 & 0.00385 & -0.15642 & -0.16027 & 15 & For t 2 & Plant & Major \\
\hline $\begin{array}{l}\text { Zn-containing } \\
\text { dehydrogenase }\end{array}$ & AF029 & 0.02937 & -0.01838 & -0.04775 & 5 & can a 1 & Fungi & Major \\
\hline
\end{tabular}

List from A to Z, Significant differential expressed AllFams supported by FDR $<0,05$ are higlighted bold, NA means not avaiable information

overlapping disease responses. In the protein sequence database which includes 509 non-redundant food allergens from fungi, plants and animals we detected 121 different allergens belonging to 74 allergen families following recognized classificatory criteria.

\section{Additional files}

Additional file 1: Phylogenetic relationships of the considered populations as an individual's selection basis for RNA sequencing of $A$. simplex s.S., A. pegreffii and their hybrid haplotype. Tree was obtained based on maximum likelihood (ML) and Bayesian inference analysis using mitochondrial COIl gene and the GTR + I + G evolutionary model. Numbers at nodes correspond to ML bootstrap proportions (BP) (above number) and Bayesian posterior probabilities (BPP) (under number). (PNG $621 \mathrm{~kb}$ )

Additional file 2: List of species and specimen used in the phylogenetic tree of Additional file 1. Code of the voucher specimen and accession number for mitochondrial gene COll (*: sequences obtained from GenBank). Labeled are the specimens selected for RNA sequencing (first number, population; second number specimen). A. simplex s.s. - A. pegreffii refers to hybrids haplotype according Abollo et al. [23]. (DOCX $47 \mathrm{~kb})$

Additional file 3: Differential expression analyses at the whole transcriptome level. Results obtained from the analyses of differential expression; 1) Hybrids vs A. pegreffi; 2) Hybrids vs A. simplex s.s.; 3) A. pegreffii vs $A$. simplex s.s. Rows summarizing significant differentially expressed transcripts supported by FDR $<0.05$ ) are highlighted in light blue. (XLSX $12115 \mathrm{~kb}$ )

Additional file 4: Differentially enriched metabolic pathways and GOs. Annotation of metabolic pathways and and GOs integrating results of differential enrichment using GOseq. (XLSX 232 kb)

Additional file 5: Differential expression of ES transcripts. 1) Count file used as input in the differential expression test performed at the ES gene level; 2) Summarization of the 356 ES genes accompanied by logFC values and their FDR support obtained from the 3 performed differential expression analyses and additional information relative to consensus sequences grouped to each gene description, as well as associated GOs terms and identification of SignalP domain. This summarization is also available in AnisakisDB as a dynamic table from where sequences and annotations can be navigated and retrieved. (XLSX $91 \mathrm{~kb}$ )
Additional file 6: Anisakis allergome. 1) Count file and results of differential expression for the 74 allergen families detected in A. simplex S.S., A. pegreffi and hybrids. 2) Blast results obtained in the search performed using 509 known allergens as queries against the reference Anisakis transcriptome. Nine hundred and thirty seven consensus sequences of the reference transcriptome were detected to significantly match to the queries and were classified into 74 allergen families of which we include additional information. This summarization is also available in AnisakisDB as a dynamic table from where sequences and annotations can be easily navigated and retrieved. (XLS $351 \mathrm{~kb}$ )

\section{Abbreviations}

AllFam : Allergen family; BCV: Biological coefficient of variation; BIC: Bayesian information criterion; BPP: Bayesian posterior probability; COll: Cytochrome oxidase subunit II; CPM: Counts per million; EC: Enzyme commission; ENA: European Nucleotide Archive; ES: Excretory/secretory; FDR: False discovery rate; Gl: Entrez Gene Identifier; GO: Gene Ontology; hybrid: Hybrid haplotype; IgE: Immunoglobulin E; ITS: Internal transcribed spacers; KOG: Eukaryotic orthologous groups; L3: Third-stage larvae; logFC: Log2 fold change; MDS: Multidimensional scaling; ML: Maximum likelihood; NR: NCBI non-redundant protein database; NT: NCBI nucleotide database; ORF: Open reading frame; TM: Transmembrane domain

\section{Acknowledgments}

We thank Rafael Capilla for his valuable contribution when constructing the AnisakisDB. Comments and suggestions of two reviewers notably improves the manuscript.

\section{Funding}

This work was supported by Spanish National project AGL2009-2485-C03-02 and partially funded by European Union project PARASITE (FP7-312068).

\section{Availability of data and materials}

COll sequences have been deposited at European Nucleotide Archive (ENA) under the accession numbers LT883269 - LT883368 (For more details, see the section AnisakisDB in Methods). Raw read data have been deposited at the NCBI SRA Study SRP072976, BioProject PRJNA316941 and BioSamples SAMN04592605, SAMN04592630, and SAMN04592599. Assemblies, sequences, annotations and backups of all analyses performed in this study are freely available in AnisakisDB at www.anisakis.mncn.csic.es.

\section{Authors' contributions}

AN, LR, SCA, MB, IM, MGM, MC, JPA and CL conceived and designed the study. AN and JPA coordinated the study. SCA, NCS, LR, RR, YJR, MC and MGM performed the biological and taxonomic experiments based on COII signal. LR, SCA, NCS and RR, performed the RNAseq experiments. CL, LR, SC, 
SCA, YJR, RR and AN performed the bioinformatics analyses. RF, CL, and AN created the database. $C L, A N, B S, L R, S C A, M B, I M, M G M, M C$ analyzed and interpreted the data. $\mathrm{CL}, \mathrm{AN}$ and $\mathrm{MB}$ wrote the manuscript. All authors read and approved the final manuscript.

\section{Competing interest}

The authors declare that they have no competing interests.

\section{Ethics approval and consent to participate}

Not applicable. Anisakis samples were collected during a survey of the FAO 27 area in the fish central Madrid Market. No ethical approval or field permissions were necessary.

\section{Consent for publication}

Not applicable.

\section{Publisher's Note}

Springer Nature remains neutral with regard to jurisdictional claims in published maps and institutional affiliations.

\section{Author details}

'Biotechvana, Scientific Park, University of Valencia, Calle Catedrático José Beltrán 2, 46980 Paterna, Valencia, Spain. ${ }^{2}$ Departamento Biodiversidad y Biología Evolutiva, Museo Nacional de Ciencias Naturales, CSIC, Calle José Gutiérrez Abascal, 2, 28006 Madrid, Spain. ${ }^{3}$ Unidad de Genómica, Campus de Cantoblanco, Scientific Park of Madrid, Calle Faraday, 7, Campus de Cantoblanco, 28049 Madrid, Spain. ${ }^{4}$ Unidad de Proteomica Centro Nacional de Biotecnología, CSIC, Calle Darwin, 3, 28049 Madrid, Spain. ${ }^{5}$ Instituto de Ciencia y Tecnología de Alimentos y Nutrición (ICTAN), Calle José Antonio Novais, 10, 28040 Madrid, Spain. ${ }^{6}$ Servicio de Immunología, Hospital Universitario La Paz, Paseo de la Castellana, 261, 28046 Madrid, Spain. ${ }^{7}$ Edinburgh Genomics, and Institute of Evolutionary Biology, School of Biological Sciences, The King's Buildings, The University of Edinburgh, Edinburgh EH9 3JT, UK.

Received: 21 May 2018 Accepted: 30 July 2018

Published online: 07 August 2018

\section{References}

1. De Ley P, Blaxter M. A new system for Nematoda: combining morphological characters with molecular trees, and translating clades into ranks and taxa. In: Cook R, Hunt DJ, editors. Nematology Monographs and Perspectives, vol. 2. Leiden-Boston: Brill; 2004. p. 633-53.

2. Van Thiel PH, Kuipers FC, Roskman RT. A nematode parasitic to herring causing acute abdominal syndromes in man. Trop Geogr Med. 1960;12:97-113.

3. CDC [http://www.cdc.gov/parasites/anisakiasis/faqs.html].

4. Audicana MT, Kennedy MW. Anisakis simplex: from Obscure Infectious Worm to Inducer of Immune Hypersensitivity. Clin Microbiol Rev. 2008;21(2): 360-79. table of contents

5. Montoro A, Perteguer MJ, Chivato T, Laguna R, Cuellar C. Recidivous acute urticaria caused by Anisakis simplex. Allergy. 1997;52(10):985-91.

6. Audicana MT, Ansotegui IJ, de Corres LF, Kennedy MW. Anisakis simplex: dangerous--dead and alive? Trends Parasitol. 2002;18(1):20-5.

7. Moneo I, Caballero ML, Gonzalez-Munoz M, Rodriguez-Mahillo Al, Rodriguez-Perez R, Silva A. Isolation of a heat-resistant allergen from the fish parasite Anisakis simplex. Parasitol Res. 2005;96(5):285-9.

8. Audicana MT, Fernandez de Corres L, Munoz D, Fernandez E, Navarro JA, del Pozo MD. Recurrent anaphylaxis caused by Anisakis simplex parasitizing fish. J Allergy Clin Immunol. 1995;96(4):558-60

9. Caballero ML, Moneo I. Several allergens from Anisakis simplex are highly resistant to heat and pepsin treatments. Parasitol Res. 2004;93(3):248-51.

10. Nieuwenhuizen N, Lopata AL, Jeebhay MF, Herbert DR, Robins TG, Brombacher F. Exposure to the fish parasite Anisakis causes allergic airway hyperreactivity and dermatitis. J Allergy Clin Immunol. 2006;117(5):1098-105.

11. Shweiki E, Rittenhouse DW, Ochoa JE, Punja VP, Zubair MH, Baliff JP. Acute Small-Bowel Obstruction From Intestinal Anisakiasis After the Ingestion of Raw Clams; Documenting a New Method of Marine-to-Human Parasitic Transmission. Open Forum Infect Dis. 2014; (1, 2):ofu087.

12. Puente P, Anadon AM, Rodero M, Romaris F, Ubeira FM, Cuellar C. Anisakis simplex: the high prevalence in Madrid (Spain) and its relation with fish consumption. Exp Parasitol. 2008;118(2):271-4.
13. Radauer C, Bublin M, Wagner S, Mari A, Breiteneder H. Allergens are distributed into few protein families and possess a restricted number of biochemical functions. J Allergy Clin Immunol. 2008;121(4):847-852 e7.

14. Radauer C, Nandy A, Ferreira F, Goodman RE, Larsen JN, Lidholm J, Pomes A, Raulf-Heimsoth M, Rozynek P, Thomas WR, et al. Update of the WHO/IUIS allergen nomenclature database based on analysis of allergen sequences. Allergy. 2014;69(4):413-9.

15. Arcos SC, Ciordia S, Roberston L, Zapico I, Jimenez-Ruiz Y, Gonzalez-Munoz M, Moneo I, Carballeda-Sangiao N, Rodriguez-Mahillo A, Albar JP, et al. Proteomic profiling and characterization of differential allergens in the nematodes Anisakis simplex sensu stricto and A. Pegreffii. Proteomics. 2014; 14(12):1547-68.

16. Baird FJ, Su X, Aibinu I, Nolan MJ, Sugiyama H, Otranto D, Lopata AL, Cantacessi $C$. The Anisakis transcriptome provides a resource for fundamental and applied studies on allergy-causing parasites. PLoS Negl Trop Dis. 2016;10(7):e0004845.

17. Hartwich G. Die Vorderarmstrukturen, das Exkretionssytem sowie der Kopfbau der Ascariden und ihre taxonomische Bedeutung. Wissenschaftliche Martin-Luther-Universitaet Halle-Wittenberg Mathematish Naturwissenschaftliche Reihe. 1954;3:1171-212.

18. Hartwich G. Zur Systematik der Nematoden-Superfamilie Ascaridoidea. Zoologische Jahrbücher Abteilung für Systematik, Ökologie und Geographie der Tier. 1957;85:211-52.

19. Fagerholm HP. Systematic implications of male caudal morphology in ascaridoid nematode parasites. Syst Parasitol. 1991;19(3):215-28.

20. Mattiucci S, Nascetti G. Advances and trends in the molecular systematics of anisakid nematodes, with implications for their evolutionary ecology and host-parasite co-evolutionary processes. In: Rollison S, editor. Advances in parasitology, vol. 66. London: Academic Press; 2004. p. 47-148.

21. Umehara A, Kawakami Y, Ooi HK, Uchida A, Ohmae H, Sugiyama H. Molecular identification of Anisakis type I larvae isolated from hairtail fish off the coasts of Taiwan and Japan. Int J Food Microbiol. 2010;143(3):161-5.

22. Martin-Sanchez J, Artacho-Reinoso ME, Diaz-Gavilan M, Valero-Lopez A. Structure of Anisakis simplex s.L. populations in a region sympatric for a. Pegreffii and A. Simplex s.S. Absence of reproductive isolation between both species. Mol Biochem Parasitol. 2005;141(2):155-62.

23. Abollo E, Paggi L, Pascual S, D'Amelio S. Occurrence of recombinant genotypes of Anisakis simplex s.S. And Anisakis pegreffii (Nematoda: Anisakidae) in an area of sympatry. Infect Genet Evol. 2003;3(3):175-81.

24. Mattiucci S, Cipriani P, Webb SC, Paoletti M, Marcer F, Bellisario B, Gibson DI, Nascetti G. Genetic and morphological approaches distinguish the three sibling species of the Anisakis simplex species complex, with a species designation as Anisakis berlandi n. Sp. for a. Simplex sp. C (Nematoda: Anisakidae). J Parasitol. 2014;100(2):199-214.

25. Mattiucci S, Nascetti G. Molecular systematics, phylogeny and ecology of anisakid nematodes of the genus Anisakis Dujardin, 1845: an update. Paraste. 2006;13(2):99-113.

26. Mattiucci S, Paoletti M, Webb SC. Anisakis nascettii n. Sp. (Nematoda: Anisakidae) from beaked whales of the southern hemisphere: morphological description, genetic relationships between congeners and ecological data. Syst Parasitol. 2009;74(3):199-217.

27. Cavallero S, Nadler SA, Paggi L, Barros NB, D'Amelio S. Molecular characterization and phylogeny of anisakid nematodes from cetaceans from southeastern Atlantic coasts of USA, Gulf of Mexico, and Caribbean Sea. Parasitol Res. 2011;108(4):781-92.

28. Nadler SA, D'Amelio S, Dailey MD, Paggi L, Siu S, Sakanari JA. Molecular phylogenetics and diagnosis of Anisakis, Pseudoterranova, and Contracaecum from northern Pacific marine mammals. J Parasitol. 2005: 91(6):1413-29.

29. Umehara A, Kawakami Y, Araki J, Uchida A. Molecular identification of the etiological agent of the human anisakiasis in Japan. Parasitol Int. 2007;56(3):211-5

30. Arizono N, Yamada M, Tegoshi T, Yoshikawa M. Anisakis simplex sensu stricto and Anisakis pegreffii: biological characteristics and pathogenetic potential in human anisakiasis. Foodborne Pathog Dis. 2012;9(6):517-21.

31. Fumarola L, Monno R, lerardi E, Rizzo G, Giannelli G, Lalle M, Pozio E. Anisakis pegreffi etiological agent of gastric infections in two Italian women. Foodborne Pathog Dis. 2009;6(9):1157-9.

32. Mattiucci S, Paoletti M, Borrini F, Palumbo M, Palmieri RM, Gomes V, Casati A, Nascetti G. First molecular identification of the zoonotic parasite Anisakis pegreffii (Nematoda: Anisakidae) in a paraffin- 
embedded granuloma taken from a case of human intestinal anisakiasis in Italy. BMC Infect Dis. 2011;11:82.

33. Mattiucci S, Fazii P, De Rosa A, Paoletti M, Megna AS, Glielmo A, De Angelis M, Costa A, Meucci C, Calvaruso V, et al. Anisakiasis and gastroallergic reactions associated with Anisakis pegreffii infection, Italy. Emerg Infect Dis. 2013;19(3):496-9.

34. Valero A, Lopez-Cuello M, Benítez R, Adroher FJ. Anisakis spp. in European hake, Merluccius merluccius (L.) from the Atlantic off northWest Africa and the Mediterranean off southern Spain. Acta Parasitol. 2006;51:209-12.

35. Abattouy N, Valero A, Lozano J, Barón SD, Romero C, Martín-Sánchez J. Population genetic analysis of Anisakis simplex s.L. and Anisakis pegreffii (Nematoda, Anisakidae) from parapatric areas and their contact zone. Parasite Epidemiol Control. 2016;1(2):169-76.

36. Umehara A, Kawakami Y, Matsui T, Araki J, Uchida A. Molecular identification of Anisakis simplex sensu stricto and Anisakis pegreffii (Nematoda: Anisakidae) from fish and cetacean in Japanese waters. Parasitol Int. 2006; 55(4):267-71.

37. Iglesias L, Valero A, Adroher FJ. Some factors which influence the in vitro maintenance of Anisakis simplex (Nematoda). Folia Parasitol. 1997; 44(4):297-301.

38. Zhu XQ, Podolska M, Liu JS, Yu HQ, Chen HH, Lin ZX, Luo CB, Song HQ, Lin $\mathrm{RQ}$. Identification of anisakid nematodes with zoonotic potential from Europe and China by single-strand conformation polymorphism analysis of nuclear ribosomal DNA. Parasitol Res. 2007;101(6):1703-7.

39. D'Amelio S, Mathiopoulos KD, Santos CP, Pugachev ON, Webb SC, Picanco M, Paggi L. Genetic markers in ribosomal DNA for the identification of members of the genus Anisakis (Nematoda: ascaridoidea) defined by polymerase-chain-reaction-based restriction fragment length polymorphism. Int J Parasitol. 2000;30(2):223-6.

40. Nadler SA, Hudspeth DS. Phylogeny of the ascaridoidea (nematoda: ascaridida) based on three genes and morphology: hypotheses of structural and sequence evolution. J Parasitol. 2000;86(2):380-93.

41. Benson DA, Karsch-Mizrachi I, Lipman DJ, Ostell J, Sayers EW. GenBank Nucleic Acids Res. 2010;38(Database issue):D46-51.

42. Thompson JD, et al. CLUSTAL W: improving the sensitivity of progressive multiple sequence alignment through sequence weighting, position-specific gap penalties and weight matrix choice. Nucleic Acids Res. 1994;22:4673-80.

43. Hall T. Bioedit: a user-friendly biological sequence alignment editor and analysis program for windows 95/98/NT. Nucleic Acids Symp Ser. 1999; 41:95-8.

44. Ronquist F, Teslenko M, van der Mark P, Ayres DL, Darling A, Hohna S, Larget B, Liu L, Suchard MA, Huelsenbeck JP. MrBayes 3.2: efficient Bayesian phylogenetic inference and model choice across a large model space. Syst Biol. 2012;61(3):539-42.

45. Darriba D, Taboada GL, Doallo R, Posada D. jModelTest 2: more models, new heuristics and parallel computing. Nat Methods. 2012;9(8):772.

46. Guindon S, Dufayard JF, Lefort V, Anisimova M, Hordijk W, Gascuel O. New algorithms and methods to estimate maximum-likelihood phylogenies: assessing the performance of PhyML 3.0. Syst Biol. 2010;59(3):307-21.

47. Rambaut A, Drummond AJ, Xie D, Baele G and Suchard MA. Tracer v1.6, 2013. Available from http://tree.bio.ed.ac.uk/software/tracer.

48. Base Space [http://basespace.illumina.com].

49. FastQC [http://www.bioinformatics.babraham.ac.uk/projects/fastqc].

50. Cutadapt MM. Removes Adapter Sequences From High-Throughput Sequencing Reads. EMBnetjournal. 2011;17(1)

51. Schmieder R, Edwards R. Quality control and preprocessing of metagenomic datasets. Bioinformatics. 2011;27(6):863-4.

52. Schulz MH, Zerbino DR, Vingron M, Birney E. Oases: robust de novo RNA-seq assembly across the dynamic range of expression levels. Bioinformatics. 2012;28(8):1086-92.

53. Zerbino DR, Birney E. Velvet: algorithms for de novo short read assembly using de Bruijn graphs. Genome Res. 2008;18(5):821-9.

54. Assemblathon_Stats [http://korflab.ucdavis.edu/datasets/Assemblathon/ Assemblathon2/Basic_metrics/assemblathon_stats.pl].

55. Altschul SF, Gish W, Miller W, Myers EW, Lipman DJ. Basic local alignment search tool. J Mol Biol. 1990;215(3):403-10.

56. Tatusov RL, Fedorova ND, Jackson JD, Jacobs AR, Kiryutin B, Koonin EV, Krylov DM, Mazumder R, Mekhedov SL, Nikolskaya AN, et al. The COG database: an updated version includes eukaryotes. BMC Bioinform. 2003;4:41.
57. Huntley RP, Sawford T, Mutowo-Meullenet P, Shypitsyna A, Bonilla C, Martin MJ, O'Donovan C. The GOA database: gene ontology annotation updates for 2015. Nucleic Acids Res. 2015;43:D1057-63.

58. Bairoch A. The ENZYME database in 2000. Nucleic Acids Res. 2000;28(1):304-5.

59. Hunter S, Apweiler R, Attwood TK, Bairoch A, Bateman A, Binns D, Bork P, Das $U$, Daugherty L, Duquenne L, et al. InterPro: the integrative protein signature database. Nucleic Acids Res. 2009;37(Database issue):D211-5.

60. Rice P, Longden I, Bleasby A. EMBOSS: the European molecular biology open software suite. Trends Genet. 2000;16(6):276-7.

61. OrfPredictor [http://proteomics.ysu.edu/tools/OrfPredictor.html].

62. Petersen TN, Brunak S, von Heijne G, Nielsen H. SignalP 4.0: discriminating signal peptides from transmembrane regions. Nat Methods. 2011;8(10):785-6.

63. Krogh A, Larsson B, von Heijne G, Sonnhammer EL. Predicting transmembrane protein topology with a hidden Markov model: application to complete genomes. J Mol Biol. 2001;305(3):567-80.

64. Kotera M, Hirakawa M, Tokimatsu T, Goto S, Kanehisa M. The KEGG databases and tools facilitating omics analysis: latest developments involving human diseases and pharmaceuticals. Methods Mol Biol. 2012;802:19-39.

65. AMOS Package [http://sourceforge.net/projects/amos].

66. Fu L, Niu B, Zhu Z, Wu S, Li W. CD-HIT: accelerated for clustering the nextgeneration sequencing data. Bioinformatics. 2012;28(23):3150-2.

67. Langmead B, Trapnell C, Pop M, Salzberg SL. Ultrafast and memory-efficient alignment of short DNA sequences to the human genome. Genome Biol. 2009;10(3):R25.

68. Davidson NM, Oshlack A. Corset: enabling differential gene expression analysis for de novo assembled transcriptomes. Genome Biol. 2014;15(7):410.

69. Robinson MD, McCarthy DJ, Smyth GK. EdgeR: a Bioconductor package for differential expression analysis of digital gene expression data. Bioinformatics. 2010;26(1):139-40.

70. Benjamini $Y$, Hochberg Y. Controlling the false discovery rate: a practical and powerful approach to multiple testing. J R Stat Soc. 1995; Series B(57):289-300.

71. Futami R, Munoz-Pomer L, Viu JM, Dominguez-Escriba L, Covelli L, Bernet GP, Sempere JM, Moya A, Llorens C. GPRO: The professional tool for management, functional analysis and annotation of omic sequences and databases. Biotechvana Bioinform: 2011-SOFT3. 2011;

72. Young MD, Wakefield MJ, Smyth GK, Oshlack A. Gene ontology analysis for RNA-seq: accounting for selection bias. Genome Biol. 2010;11(2):R14.

73. The UniProt Consortium. UniProt: the universal protein knowledgebase. Nucleic Acids Res. 2017;45(D1):D158-69.

74. P H P programming language [http://php.net].

75. MySQL [http://www.mysql.com].

76. Apache [http://www.apache.org]

77. Altschul SF, Madden TL, Schaffer AA, Zhang J, Zhang Z, Miller W, Lipman DJ. Gapped BLAST and PSI-BLAST: a new generation of protein database search programs. Nucleic Acids Res. 1997;25(17):3389-402.

78. Ernst S, Langer R, Cooney CL, Sasisekharan R. Enzymatic Degradation of Glycosaminoglycans. Crit Rev Biochem Mol Biol. 1995:30(5).

79. Shears SB, Ganapathi SB, Gokhale NA, et al. Defining Signal Transduction by Inositol Phosphates. In: Balla T, Wymann M, York JD, editors.

Phosphoinositides II: The Diverse Biological Functions. Netherlands: Springer; 2012. p. 389-412.

80. Feng $L$, Shou Q, Butcher RA. Identification of a dTDP-rhamnose biosynthetic pathway that oscillates with the molting cycle in Caenorhabditis elegans. Biochem J. 2016:473(11):1507-21.

81. Braeckman BP, Houthoofd K, Vanfleteren JR. Intermediary metabolism. In: WormBook, ed. The C. elegans Research Community, WormBook. https:// doi.org/10.1895/wormbook.1.146.1. 2009 (http://www.wormbook.org).

82. Wolkow CA. Regulation of invertebrate longevity by inositol phosphate signaling. Advan Cell Aging Gerontol. 2003;12:27-46.

83. McElwee JJ, Schuster E, Blanc E, Thornton J, Gems D. Diapause-associated metabolic traits reiterated in long-lived daf-2 mutants in the nematode Caenorhabditis elegans. Mech Ageing Dev. 2006;127(5):458-72.

84. Holt SJ, Riddle DL. SAGE surveys C. elegans carbohydrate metabolism: evidence for an anaerobic shift in the long-lived dauer larva. Mech Ageing Dev. 2003;124(7):779-800.

85. Burnell AM, Houthoofd K, O'Hanlon K, Vanfleteren JR. Alternate metabolism during the dauer stage of the nematode Caenorhabditis elegans. Exp Gerontol. 2005;40(11):850-6.

86. Gilabert A, Wasmuth JD. Unravelling parasitic nematode natural history using population genetics. Trends Parasitol. 2013;29(9):438-48. 
87. Cipriani P, Smaldone G, Acerra V, D'Angelo L, Anastasio A, Bellisario B, Palma G, Nascetti G, Mattiucci S. Genetic identification and distribution of the parasitic larvae of Anisakis pegreffii and Anisakis simplex (s. s.) in European hake Merluccius merluccius from the Tyrrhenian Sea and Spanish Atlantic coast: implications for food safety. Int J Food Microbiol. 2015;198:1-8.

88. Karl H. Nematode larvae in fish on the German market: 20 years of consumer related research. Arch Leb. 2008:59:107-16.

89. Karl H, Meyer C, Banneke S, Jark U, Feldhusen F. The abundance of nematode larvae Anisakis sp. in the flesh of fishes and possible post mortem migration. Arch Leb. 2002;53:118-20.

90. Quiazon KM, Yoshinaga T, Ogawa K. Experimental challenge of Anisakis simplex sensu stricto and Anisakis pegreffii (Nematoda: Anisakidae) in rainbow trout and olive flounder. Parasitol Int. 2011;60(2):126-31.

91. Smith JW. The abundance of Anisakis simplex $L 3$ in the body-cavity and flesh of marine teleosts. Int J Parasitol. 1984;14:491-5.

92. Hauck AK. Occurrence and survival of the larval nematode Anisakis sp. in the flesh of fresh, frozen, brined, and smoked pacific herring, Clupea harengus pallasi. J Parasitol. 1977;63(3):515-9.

93. Smith JW, Wootten R. Experimental studies on the migration of Anisakis sp. larvae (Nematoda: ascaridida) into the flesh of herring, Clupea harengus $L$. Int J Parasitol. 1975;5(2):133-6.

94. Ciprianl P, Acerra V, Bellisario B, Sbaraglia GL, Cheleschi R, Nascetti G, Mattiucci S. Larval migration of the zoonotic parasite Anisakis pegreffi (Nematoda: Anisakidae) in European anchovy, Engraulis encrasicolus: implications to seafood safety. Food Control. 2016;59:148-57.

95. Bahlool QZ, Skovgaard A, Kania PW, Buchmann K. Effects of excretory/ secretory products from Anisakis simplex (Nematoda) on immune gene expression in rainbow trout (Oncorhynchus mykiss). Fish Shellfish Immunol. 2013;35(3):734-9.

96. Coscia MR, Oreste U. Presence of antibodies specific for proteins of Contracaecum osculatum (Rudolphi, 1908) in plasma of several Antarctic teleosts. Fish Shellfish Immunol. 1998;8:295-302.

97. Marsh DG. Allergens and the genetics of allergy. In: Sela III M, editor. The Antigens. New York: Academic Press; 1975. p. 271-350.

98. Lowenstein H. Quantitative immunoelectrophoretic methods as a tool for the analysis and isolation of allergens. Progress in Allergy. 1978;25:1-62.

99. Sellers JR. Myosins: a diverse superfamily. Biochim Biophys Acta. 2000; 1496(1):3-22.

100. Tsai LC, Peng HJ, Lee CS, Chao PL, Tang RB, Tsai JJ, Shen HD, Hung MW Han SH. Molecular cloning and characterization of full-length cDNAs encoding a novel high-molecular-weight Dermatophagoides pteronyssinus mite allergen, Der p 11. Allergy. 2005;60(7):927-37.

101. Jeong KY, Hong CS, Yong TS. Allergenic tropomyosins and their crossreactivities. Protein Pept Lett. 2006;13(8):835-45.

102. Rodriguez-Mahillo Al, Gonzalez-Munoz M, Gomez-Aguado F, RodriguezPerez R, Corcuera MT, Caballero ML, Moneo I. Cloning and characterisation of the Anisakis simplex allergen Ani s 4 as a cysteine-protease inhibitor. Int J Parasitol. 2007;37(8-9):907-17.

103. Kobayashi Y, Ishizaki S, Shimakura K, Nagashima Y, Shiomi K. Molecular cloning and expression of two new allergens from Anisakis simplex. Parasitol Res. 2007;100(6):1233-41.

104. Kobayashi Y, Shimakura K, Ishizaki S, Nagashima Y, Shiomi K. Purification and cDNA cloning of a new heat-stable allergen from Anisakis simplex. Mol Biochem Parasitol. 2007;155(2):138-45.

105. Rodriguez-Perez R, Moneo I, Rodriguez-Mahillo A, Caballero ML. Cloning and expression of Ani s 9, a new Anisakis simplex allergen. Mol Biochem Parasitol. 2008;159(2):92-7.

106. Caballero ML, Umpierrez A, Moneo I, Rodriguez-Perez R. Ani s 10, a new Anisakis simplex allergen: cloning and heterologous expression. Parasitol Int. 2011;60(2):209-12.

107. Kobayashi Y, Kakemoto S, Shimakura K, Shiomi K. Molecular cloning and expression of a new major allergen, Ani s 14, from Anisakis simplex. Shokuhin Eiseigaku Zasshi. 2015;56(5):194-9.

108. Carballeda-Sangiao N, Rodriguez-Mahillo Al, Careche M, Navas A, Caballero T, Dominguez-Ortega J, Jurado-Palomo J, Gonzalez-Munoz M. Ani s 11-like protein is a pepsin- and heat-resistant major allergen of Anisakis spp. and a valuable tool for Anisakis allergy component-resolved diagnosis. Int Arch Allergy Immunol. 2016;169(2):108-12.

109. Arrieta I, del Barrio M, Vidarte L, del Pozo V, Pastor C, Gonzalez-Cabrero J, Cardaba B, Rojo M, Minguez A, Cortegano I, et al. Molecular cloning and characterization of an IgE-reactive protein from Anisakis simplex: Ani s 1. Mol Biochem Parasitol. 2000;107(2):263-8.

110. Cuellar CG-FJ, Rodero M, Valls A, de Frutos C, Daschner A, Nieuwenhuizen N. La Hemoglobina de Anisakis simplex: factores que influyen sobre su reconcimiento. In: XVIII Congreso de la Sociedad Espanola de Parasitologia; $63 \%$ of patients allergenicity 2013; 2013.

111. Helton da Costa Santiago SB, Ribeiro JMC, Nutman TB. Structural Differences between Human Proteins and Aero- and Microbial Allergens Define Allergenicity. PLoS One. 2012;7:e40552.

112. Lopez I, Pardo MA. A phage display system for the identification of novel Anisakis simplex antigens. J Immunol Methods. 2011;373(1-2):247-51.

113. Faeste CK, Jonscher KR, Dooper MM, Egge-Jacobsen W, Moen A, Daschner A, Egaas E, Christians U. Characterisation of potential novel allergens in the fish parasite Anisakis simplex. EuPA Open Proteom. 2014;4:140-55.

114. Cavallero S, Lombardo F, Su X, Salvemini M, Cantacessi C, D'Amelio S. Tissue-specific transcriptomes of Anisakis simplex (sensu stricto) and Anisakis pegreffii reveal potential molecular mechanisms involved in pathogenicity. Parasit Vectors. 2018;11:31.

115. PARASITE Parasite risk assessment with integrated tools in EU fish production value chains (EU Grant agreement no.: 312068). https://cordis. europa.eu/result/rcn/189395_en.html.

116. Alonso-Gomez A, Moreno-Ancillo A, Lopez-Serrano MC, Suarez-de-Parga JM, Daschner A, Caballero MT, Barranco P, Cabanas R. Anisakis simplex only provokes allergic symptoms when the worm parasitises the gastrointestinal tract. Parasitol Res. 2004;93(5):378-84.

117. Baeza ML, Rodriguez A, Matheu V, Rubio M, Tornero P, de Barrio M, Herrero T, Santaolalla M, Zubeldia JM. Characterization of allergens secreted by Anisakis simplex parasite: clinical relevance in comparison with somatic allergens. Clin Exp Allergy. 2004;34(2):296-302.

118. Pascual CY, Crespo JF, San Martin S, Ornia N, Ortega N, Caballero T, Munoz-Pereira M, Martin-Esteban M. Cross-reactivity between IgEbinding proteins from Anisakis, German cockroach, and chironomids. Allergy. 1997;52(5):514-20.

119. Guarneri F, Guarneri C, Benvenga S. Cross-reactivity of Anisakis simplex: possible role of Ani s 2 and Ani s 3. Int J Dermatol. 2007;46(2):146-50.

\section{Ready to submit your research? Choose BMC and benefit from:}

- fast, convenient online submission

- thorough peer review by experienced researchers in your field

- rapid publication on acceptance

- support for research data, including large and complex data types

- gold Open Access which fosters wider collaboration and increased citations

- maximum visibility for your research: over $100 \mathrm{M}$ website views per year

At BMC, research is always in progress.

Learn more biomedcentral.com/submissions 